\title{
Interference mitigation using random antenna selection in millimeter wave beamforming system
}

\author{
Woochang Lim¹, Girim Kwon²* (1) and Hyuncheol Park²
}

\begin{abstract}
In multi-user beamforming systems, the inter-user interference are controlled by spatial filtering. Since the implementation of digital beamforming is difficult due to high hardware cost of large array system, the analog beamforming system with one phase shifter at each antenna element is considered with antenna selection scheme. However, the beamwidth is not sufficiently narrow to perfectly remove the interference due to limited size of antenna arrays. To mitigate the interference, we propose the random antenna selection system in millimeter wave channel. The random antenna selection expands the effective array size so that the beamwidth becomes narrow. We compare the beamwidth of conventional fixed antenna selection with random antenna selection and analyze the amount of interference for each selection scheme. Theoretical analysis and bit error rate simulation results indicate that the beamforming with random antenna selection can take advantage of large array.
\end{abstract}

Keywords: Millimeter wave, Beamforming, Antenna selection, Large array

\section{Introduction}

Millimeter wave (mmWave) is one of the leading candidates for the future wireless communications system thanks to the following two merits [1]. First, the large unlicensed bandwidth ( 30 to $300 \mathrm{GHz}$ ) allows us to solve the spectrum shortage. Second, the short wavelength of mmWave enables the devices to have a compact size with a lot of antenna elements. To utilize mmWave band, proper communication techniques suited for the channel characteristic should be employed. To this end, there are many mmWave-related standardization works such as European computer manufacturers association (ECMA)-387, IEEE 802.15.3c, IEEE 802.11ad, Wireless high-definition (WirelessHD), Next generation millimeter wave specification (NGmS), and Communication access long and medium range (CALM).

Since free-space path loss is proportional to frequency squared according to Friis equation, mmWave experiences more significant path loss than microwave below

*Correspondence: girim88@kaist.ac.kr

${ }^{2}$ School of Electrical Engineering, Korea Advanced Institute of Science and Technology (KAIST), Daehak-ro 291, Daejeon 305-338, Republic of Korea

Full list of author information is available at the end of the article
$6 \mathrm{GHz}$ [2]. Directional transmission such as beamforming is thus necessary because channel capacity and error rate depend on signal to noise ratio (SNR). Also, small wave length enables the achievement of high gain through synthesis of more antennas within limited area. However, the hardware complexity should be considered as the number of antenna elements are increased.

Maximal ratio transmission (MRT) is known as an optimal transmit beamforming technique where a dominant right singular vector of the channel matrix is used for precoding [3]. Unfortunately, high hardware complexity and cost make it difficult to implement MRT because it demands baseband digital signal processing (DSP) for each antenna element. For this reason, analog radio frequency (RF) beamforming is considered [4]. Equal gain transmission is one of the analog RF beamforming schemes controlling only phase of signal with phase shifters [5, 6]. Also, analog-digital hybrid beamforming techniques with the limited number of RF chains are considered to reduce the hardware complexity especially in mmWave beamforming systems [7-10].

In single-user transmission system, a beamforming scheme for secure communication called antenna sub- 
set modulation (ASM) has been proposed in [11]. By selecting an antenna subset randomly for every symbol, ASM randomizes the amplitude and phase of received signal along a sidelobe. Although the original purpose of ASM is to average out the signals for undesired direction by randomization, we can use the random antenna selection scheme to mitigate the inter-user interference in multi-user system which is considered in this paper. In multi-user transmission system, there exist many precoding techniques to suppress the inter-user interference. In [12], the codebook-based precoding with user scheduling algorithms has been proposed. In [13, 14], the linear methods such as block diagonalization precoding and non-linear methods such as Tomlinson-Harashima precoding are introduced. These techniques require a lot of RF chains to use DSP for each antenna element which results in high hardware complexity.

In this paper, we consider mmWave analog RF beamforming system transmitting multiple streams simultaneously with one phase shifter at each antenna element for low hardware complexity. Motivated by [11], we propose the random antenna selection scheme for multi-user downlink system and analyze the amount of inter-user interference. We compare the performance of the random antenna selection with the conventional fixed antenna selection.

In the random antenna selection, $M$ antenna elements are selected out of entire $N$ antenna elements for each user. In the fixed antenna selection, on the other hand, $M$ adjacent antenna elements are used for each user. Therefore, the effective aperture size of the random antenna selection for each user is larger than that of the fixed antenna selection. Since the beamwidth is proportional to the inverse of effective array size [15], the random antenna selection gives a narrower beamwidth. Finally, the interference with the signals for other users can be mitigated. In the massive antenna array system, we can take advantage of large effective array size by random antenna selection.

The rest of this paper is organized as follows: in Section 2, the system model and channel model are described. The beamforming techniques with antenna selection are introduced in Section 3. We give the performance analysis for the conventional fixed antenna selection and the proposed random antenna selection in Section 4. The simulation results are provided in Section 5. The Conclusion follows in Section 6.

\section{System description}

In this section, we present our system model and channel model. We consider multi-user MIMO system and mmWave channel.

\subsection{System model}

We consider a multi-user MIMO downlink system shown in Fig. 1. The base station (BS) has an antenna array with $N$ isotropic elements which have a unit gain, and each user has a single antenna. The modulated symbols are multiplied by the spread code and transmitted by analog beamforming. We assume that the analog beamforming system controls the phase of transmitted signal with phase shifters, but cannot control the amplitude of the signals. In addition, each antenna element has only one phase shifter so that the system has low hardware complexity. Therefore, $K$ exclusive subsets of antenna elements are allocated to $K \mathrm{RF}$ chains to support a multi-user transmission. A spread code is used to separate the closely located users because they cannot be perfectly separated by beamforming techniques only. Since the finite number of antenna elements cannot provide sufficiently narrow beamwidth, a spread code is needed to distinguish the overlapped users. The spread symbol at chip time $l$ which is represented by

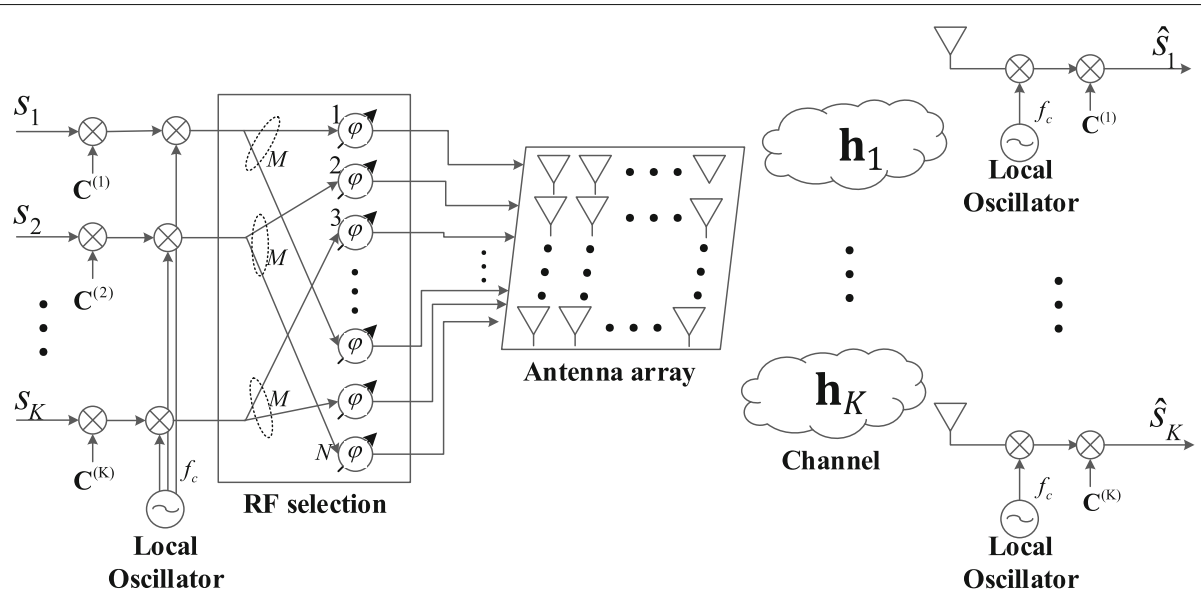

Fig. 1 Block diagram of MU-MIMO analog beamforming system 
$x_{k}[l]$ can be expressed as multiplication of the spread code $C^{(k)}[l]$ of length $L$ with the transmit symbol $s_{k}$.

$$
x_{k}[l]=C^{(k)}[l] s_{k} \text {. }
$$

In our system, we assume that the BS has the information of angle of departures and corresponding path gains to compose the beamforming weight vectors. In order to obtain such channel state information (CSI) in mmWave beamforming system with the limited number of RF chains, two types of channel estimation techniques can be considered; closed loop estimation $[16,17]$ and open loop estimation [18]. For these channel estimation techniques, the beam training period is required to obtain the CSI. In the beam training period, the BS transmits a pilot beam pattern sequence to all users. After estimating the CSI using the pilot beam pattern sequence, each user feedbacks the estimated CSI to the base station for the purpose of transmit beamforming. Since multiple signals are transmitted simultaneously, the received signal for user $m$ can be expressed by a summation of transmitted signals for all users as follows.

$$
\begin{aligned}
r_{m}[l] & =\mathbf{h}_{m}^{H}\left(\sum_{k=1}^{K} \mathbf{w}_{k} x_{k}[l]\right)+v_{m}[l] \\
& =\mathbf{h}_{m}^{H}\left(\sum_{k=1}^{K} \mathbf{w}_{k} C^{(k)}[l] s_{k}\right)+v_{m}[l], \quad \text { for } 1 \leq l \leq L,
\end{aligned}
$$

where $\mathbf{h}_{m} \in \mathbb{C}^{N \times 1}$ is channel vector for user $m$, and $\mathbf{w}_{k} \in$ $\mathbb{C}^{N \times 1}$ is the beamforming vector for user $k$ consisted of $M$ non-zero complex numbers and $N-M$ zeros. $M=\frac{N}{K}$ is the number of antenna elements allocated to each user. $v_{m}[l]$ is the complex Gaussian noise with zero mean and variance of $\sigma^{2}$.

After despreading, the received signal can be written as

$$
\begin{aligned}
\hat{r}_{m} & =\frac{1}{L} \sum_{l=1}^{L} C^{(m)}[l] \mathbf{h}_{m}^{H}\left(\sum_{k=1}^{K} \mathbf{w}_{k} C^{(k)}[l] s_{k}\right)+\frac{1}{L} \sum_{l=1}^{L} C^{(m)}[l] v_{m}[l] \\
& =\rho_{m, m} \mathbf{h}_{m}^{H} \mathbf{w}_{m} s_{m}+\sum_{\substack{k=1 \\
k \neq m}}^{K} \rho_{k, m} \mathbf{h}_{m}^{H} \mathbf{w}_{k} s_{k}+\tilde{v}_{m},
\end{aligned}
$$

where $\rho_{k, m}=\frac{1}{L} \sum_{l=1}^{L} C^{(m)}[l] C^{(k)}[l]$. We see that the interference can be mitigated by both spread code and beamforming vectors. The more beamforming mitigates the interference, the code length $L$ can be shorter.

The antenna array can be an arbitrary geometry such as linear array, rectangular array, cylindrical array, and spherical array. Since the antenna array has a sub-array structure with $K \mathrm{RF}$ chains, it should be separated into $K$ exclusive subsets. We consider two methods for antenna selection; fixed antenna selection and random antenna selection. These selection methods are presented in Section 3.

\subsection{Channel model}

In general, MIMO channel models can be separated into two categories. One is the physical model and the other is the analytical model [19]. In the physical models, we can express the physical propagation from transmit array and receive array by using the double-directional impulse response, $h\left(t, \tau, \theta^{t}, \phi^{t}, \theta^{r}, \phi^{r}\right)$, where $t$ is the observed time, $\tau$ is the delay, and $\left(\theta^{t}, \phi^{t}\right)$ and $\left(\theta^{r}, \phi^{r}\right)$ are the (elevation,azimuth) angles of departure and arrival, respectively [20]. We consider the double directional impulse response which is composed by the sum of the contributions from the discrete multi-path components, such that

$$
\begin{aligned}
h\left(t, \tau, \theta^{t}, \phi^{t}, \theta^{r}, \phi^{r}\right)= & \sum_{p=1}^{\bar{N}(t)} \bar{\rho}_{p} e^{j \psi_{p}} \delta\left(\tau-\tau_{p}\right) \delta\left(\theta^{t}-\theta_{p}^{t}\right) \\
& \times \delta\left(\phi^{t}-\phi_{p}^{t}\right) \delta\left(\theta^{r}-\theta_{p}^{r}\right) \delta\left(\phi^{r}-\phi_{p}^{r}\right),
\end{aligned}
$$

where $\bar{N}(t)$ is the number of multi-path components, and $\beta_{p} \triangleq \bar{\rho}_{p} e^{j \psi_{p}}$ is the complex path gain. The superscripts $t$ and $r$ correspond to the transmit and receive array, respectively.

The double-directional model is useful because it is independent of the antenna array geometry. However, it is hard to theoretically analyze the system. Therefore, the analytical models are considered. These models describe the channel as a transfer function whose $(i, j)$ entry is the transfer function from $j$-th transmit to the $i$-th receive antenna element. We can describe the channel transfer function matrix from the double-directional model. When the BS has $N$ antenna elements, and each of $K$ users is equipped with a single antenna, the channel vector for user- $k$ can be written as follows:

$$
\mathbf{h}_{k}=\sum_{p=1}^{\bar{N}} \bar{\rho}_{k, p} e^{j \psi_{k, p}} e^{-j 2 \pi f_{c} \tau_{k, p}} \mathbf{a}_{t}\left(\theta_{k, p}^{t}, \phi_{k, p}^{t}\right),
$$

where $p$ is the index of paths, and $f_{c}$ is carrier frequency, and $\psi_{k, p} \in[0,2 \pi]$ is uniformly distributed phase, and $\mathbf{a}_{t}$ is $N \times 1$ array steering vector. Also, we ignore the dependence on $t$ because the block fading is assumed as usual.

Radio signals are affected by many factors in their physical path [2]. The factors include the path-loss, reflection, diffraction, and scattering, and these are dependent on the signal's frequency. The mmWave suffers from the high path loss according to Friis formula, and has narrow 
shadow boundary due to small diffraction angle. Furthermore, the short wavelength causes the limited scattering which cannot make the Rayleigh scattering. As a result, the line of sight (LOS) component is dominant in mmWave channel. The measurement of LOS and non-line of sight (NLOS) component in urban outdoor mmWave channel is provided by [21]. Rappaport et al. [21] shows that there is no root-mean-square (RMS) delay spread for all LOS links in mmWave channel, and the LOS path has no resolvable multi-path. Finally, we can consider the first path of (5) as the LOS path and the others as NLOS paths. Then, we can separate the channel into a LOS component and $(\bar{N}-1)$ NLOS components as follows:

$$
\begin{aligned}
\mathbf{h}_{k}= & \bar{\rho}_{k, L O S} \mathbf{a}_{t}\left(\theta_{k, L O S}^{t}, \phi_{k, L O S}^{t}\right) \\
& +\sum_{p=2}^{\bar{N}} \bar{\rho}_{k, p} e^{j \psi_{k, p}} e^{-j 2 \pi f_{c} \tau_{k, p}} \mathbf{a}_{t}\left(\theta_{k, p}^{t}, \phi_{k, p}^{t}\right) .
\end{aligned}
$$

\section{Beamforming with antenna selection}

In this section, we introduce the beamforming techniques with antenna selection to form the multi-user transmission. Because each antenna has a single phase shifter, a subset of antenna array provides only one beam. In other words, we need to separate the entire antenna array into $K$ subsets, and consider two types of antenna selection schemes; conventional fixed antenna selection [22, 23] and random antenna selection. After selecting $K$ subsets, $K$ weight vectors are constructed as the analog beam steering vectors with directions of $K$ users.

The detail descriptions of the conventional fixed antenna selection and the proposed random antenna selection are presented in Section 3.2 and Section 3.3. In Section 4 and Section 5, we show that the random antenna selection mitigates the interference with signals for other users. Intuitively, this advantage comes from the fact that the effective array size of random antenna selection scheme is larger than that of fixed antenna selection. In other words, the beamwidth becomes narrow which decreases the amount of interference.

\subsection{Effective array size and beamwidth}

Although the spread code is used, the user- $k$ can be interfered with the signals for other users due to inaccurate synchronization or non-zero cross correlation between spread codes. The amount of interference is affected by the beamwidth overlapping with other user's beam. The beamwidth is proportional to the inverse of effective array size which is dependent on the entire array size and steering angle [15]. The effective array size is the area of antenna array projected onto the perpendicular plane to the steering angle. The projected area is presented in Fig. 2. For example, for the linear array along with y-axis,

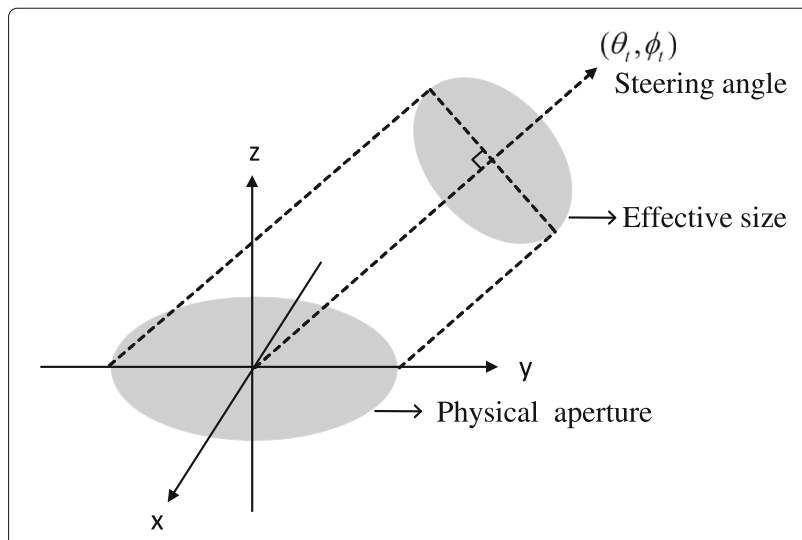

Fig. 2 Effective array size

the effective array length becomes $L \cos \theta_{t}$, where $L$ is the actual array length, and $\theta_{t}$ is the steering angle.

\subsection{Fixed antenna selection}

When the antenna array is separated into $K$ subsets, $K$ fixed patterns can be used to select the antenna elements, and each subset should be selected not to generate grating lobes which cause the strong interference with the signals for other users. Therefore, assuming that the antenna elements are spaced at half wavelength, we have to select the adjacent elements to make a subset as [22,23]. Otherwise, the grating lobes located at the fixed direction cause significant inter-user interference to certain users who are located at the same direction of the grating lobes. For example, for uniform planar array (UPA) placed on yzplane, the fixed antenna selection patterns are presented in Fig. 3. In this case, the array steering vector for UPA is given by [15],

$$
\mathbf{a}(\mathbf{k})=\left[\begin{array}{c}
1 \\
e^{-j \frac{2 \pi}{\lambda}\left(d_{z} \cos \theta\right)} \\
\vdots \\
e^{-j \frac{2 \pi}{\lambda}\left(p d_{y} \sin \theta \sin \phi+q d_{z} \cos \theta\right)} \\
\vdots \\
e^{-j \frac{2 \pi}{\lambda}\left(\left(N_{y}-1\right) d_{y} \sin \theta \sin \phi+\left(N_{z}-1\right) d_{z} \cos \theta\right)}
\end{array}\right],
$$

where $\mathbf{k}=k_{0}(\sin \theta \cos \phi, \sin \theta \sin \phi, \cos \theta)$ is wave vector, and $k_{0}=\frac{2 \pi}{\lambda}$ is wave number, and $\lambda$ is wavelength. Assuming LOS dominant channel, each element of the weight vector for user- $k$ is given by

$w_{k}(p, q)=\left\{\begin{array}{cl}\frac{1}{M} e^{j \frac{2 \pi}{\lambda}\left(p d_{y} \sin \theta_{k} \sin \phi_{k}+q d_{z} \cos \theta_{k}\right)} & \text { for }(p, q) \in \Omega_{k} \\ 0 & \text { for }(p, q) \notin \Omega_{k}\end{array}\right.$,

where $\theta_{k}$ and $\phi_{k}$ are elevation and azimuth angle of user$k$ 's position, respectively. The $N_{y}\left(N_{z}\right)$ is the number of 


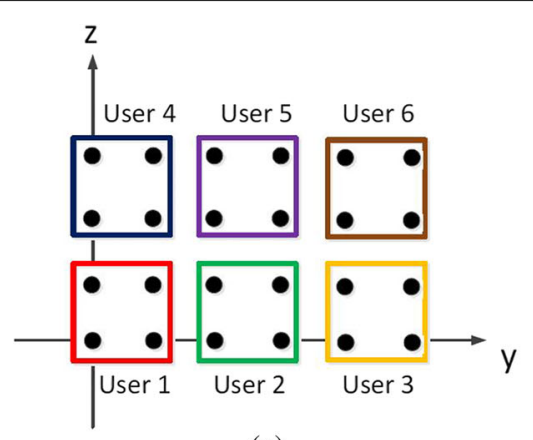

(a)

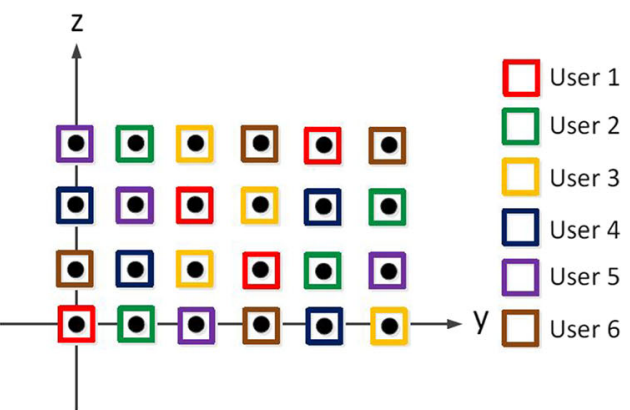

(b)

Fig. 3 The fixed and random antenna selection patterns for uniform planar array $: K=6$. a Fixed patterns. b Random patterns

antenna elements along the $y$-axis(z-axis) such that $N=$ $N_{y} N_{z}$. The $d_{y}\left(d_{z}\right)$ is the uniform antenna spacing along the $y$-axis(z-axis). The $M$ is the number of antenna elements allocated to each user. $\Omega_{k}$ is the selected antenna subset for user- $k$. Then the beam pattern for user- $k$ is obtained by inner product of array steering vector and weight vector as follows:

$$
\begin{aligned}
& B_{k}(\theta, \phi)
\end{aligned}
$$

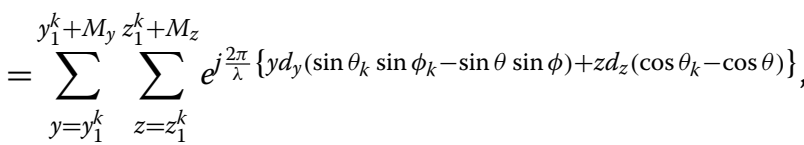

where $\left(y_{1}^{k}, z_{1}^{k}\right)$ is the first element in $\Omega_{k}=\left\{\left(y_{1}^{k}, z_{1}^{k}\right)\right.$, $\left.\left(y_{1}^{k}, z_{1}^{k}+1\right), \cdots,\left(y_{1}^{k}+M_{y}, z_{1}^{k}+M_{z}\right)\right\}$, and $M_{y}$ and $M_{z}$ are the number of elements along the $y$-axis and $z$-axis in a subset, respectively.

\subsection{Random antenna selection}

The other selection scheme considered in this paper is random selection. Every antenna element has same probability to be chosen. To provide fairness to all users, the selection is changed at symbol rate. If the antenna array elements are selected arbitrarily, the grating lobes can be formed to undesired direction. However, we can average out the grating lobes by randomness in beam patterns. In addition, the beamwidth becomes narrow because the effective array size is larger than that of fixed antenna selection. As a result, the amount of interference with the signals for other users can be decreased.

Since the beam pattern for random antenna selection cannot be determined due to its randomness, it should be analyzed by statistical model. The analysis is derived in Section 4. Instead, the beam pattern which is averaged over 100 random beam patterns is chosen to be compared with the fixed antenna selection. In Fig. 4, the comparison of beam patterns are shown. The desired direction is set to be $(\theta, \phi)=\left(120^{\circ}, 30^{\circ}\right)$. The results show that the random antenna selection gives narrower main lobes but higher side lobes than fixed antenna selection. Fortunately, since the LOS component is dominant in mmWave channel [11], the effect of increased level of side lobes which is still much lower than the level of main lobe is negligible. Therefore, the beamwidth of main lobe dominates the system performance. Note that the fixed antenna selection scheme with non-adjacent elements can also provide narrower beam than that with adjacent elements due to large effective array size. However, the antenna spacing which is larger than half-wavelength causes the grating lobes [15]. Due to the fixed position of the grating lobe, some other users who are located on the direction of the grating lobe may suffer from the significant interference. In the case of the random antenna selection scheme, on the other hand, the interference from random direction of the grating lobes are averaged out so that other users who are located on different directions can receive their desired signals.

The random antenna selection can be implemented by sorting the long pseudo random sequence generated in advance. First, we choose $N$ numbers from the sequence generated by random number generator. After sorting the chosen numbers, we allocate the $M$ numbers to each user in consecutive order. On the other hand, the fixed antenna selection need not the sorting procedure. It only needs the consecutive sequence of length $N$ to allocate the $M$ numbers to each user in consecutive order. Once the random sequence (for random selection) and consecutive sequence (for fixed selection) are generated, they can be used permanently. Therefore, the difference between random selection and fixed selection is time complexity of sorting procedure which is given by $O\left(n^{2}\right)$.

\subsection{Discussion on the case of multiple paths}

When the BS simultaneously supports the multiple users, the BS should distinguish among the paths of the users using the analog beamforming architecture. In this subsection, we discuss the possibility of user separation in our 


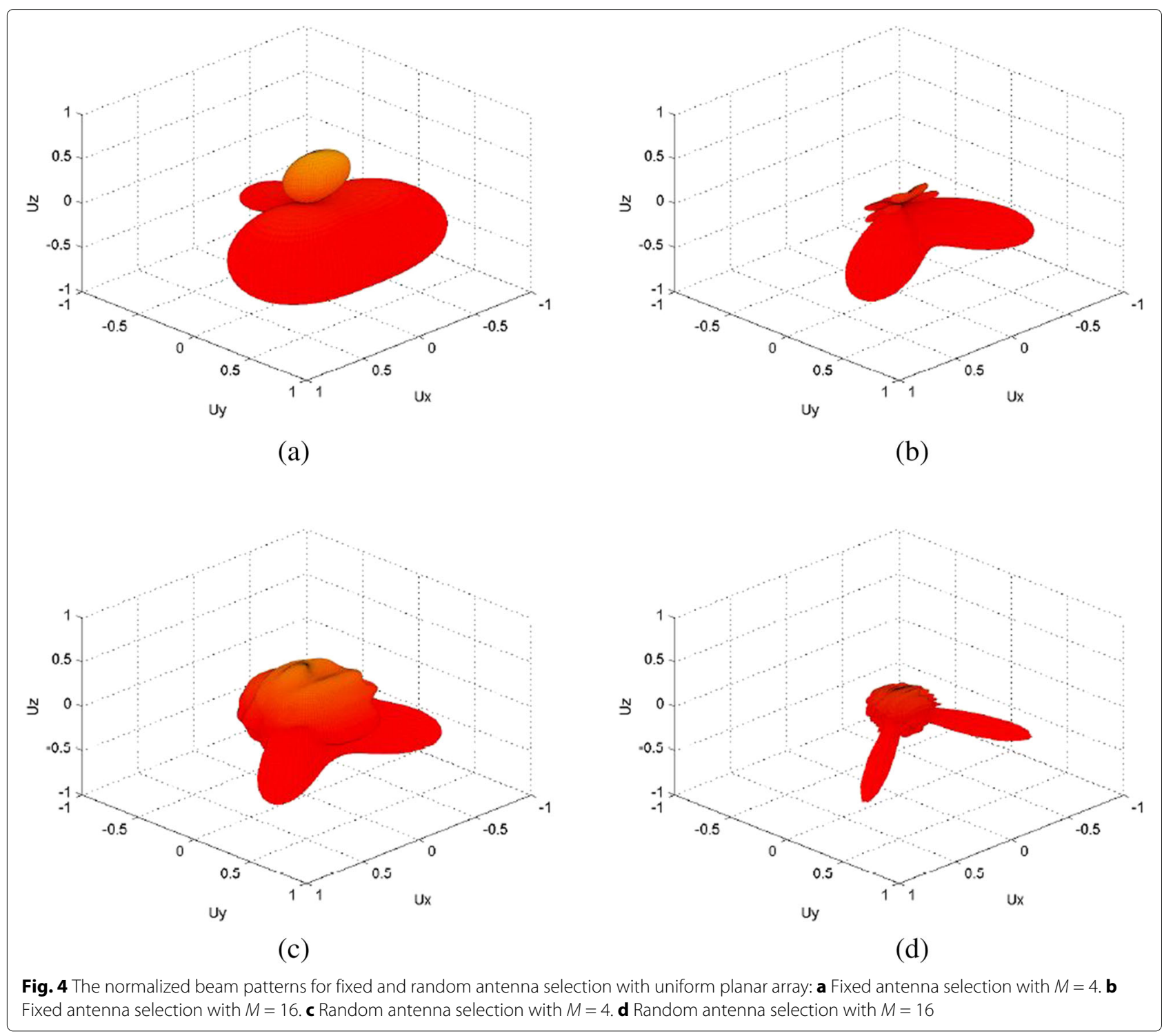

analog architecture when there exist the multiple paths in mmWave channel.

First of all, we discuss the case of LOS channel. Since the BS transmits one symbol using the analog beamforming for each user, the BS cannot manage the inter-user interference. However, if two users are properly separated in their locations, the BS can distinguish the two users by making two directional beams. Nevertheless, it is still impossible to distinguish the two users when they are closely located to each other. For this case, the spread code is used for separating the closely located users. Here, the difference from the conventional code division multiple access (CDMA) system is that the code length can be shorter than that of CDMA system due to the usage of beamforming technique.

In the case of multiple paths, the direction of the beamforming vector for each user is determined by the dominant direction in the paths, i.e., LOS path. As we will discuss in Section 5, the path loss difference between LOS and NLOS links is quite large in millimeter wave channel, e.g., $18 \mathrm{~dB}$ at $30 \mathrm{~m}$ and $21 \mathrm{~dB}$ at $50 \mathrm{~m} \mathrm{[21].} \mathrm{Although}$ multiple paths exist, the effective difference of channel gains between LOS and NLOS links becomes much larger than the difference of path loss because of the beamforming gain. Therefore, the BS can mitigate the interference by means of both beamforming gain and spread code. The effect of the multiple paths on the performance is provided in Section 5.

\section{Performance analysis}

In this section, we compare the beamwidth of each selection scheme, and the amount of interference with signals for other users are discussed. A cuboidal array is assumed for convenient analysis, and the results in this section can 
be easily applied to the uniform linear array (ULA)s or UPAs which are placed on arbitrary 1D line or 2D plane in the space. Note that since $K$ beamforming vectors are constructed as the array steering vectors for the directions of $K$ users, the system always guarantees the maximum beam gain for the desired direction. On the other hand, the interference is affected by the side lobes of the beam pattern which depend on the antenna selection pattern. As a result, only the inter-user interference is affected by the introduced randomness, and the desired signal is not changed.

\subsection{Null to null beamwidth}

To compare the beamwidth of 3D beam patterns, we define $\left(u_{x}, u_{y}, u_{z}\right)$ space in terms of directional cosines,

$$
\begin{aligned}
& u_{x} \triangleq \sin \theta \cos \phi, \\
& u_{y} \triangleq \sin \theta \sin \phi, \\
& u_{z} \triangleq \cos \theta .
\end{aligned}
$$

\subsubsection{Fixed antenna selection}

In the received signal (2), the weight vector can be expressed as

$$
w_{k}(x, y, z)=\left\{\begin{array}{cc}
\frac{1}{M} e^{j k_{0}\left(\sin \left(\theta_{k}\right) \cos \left(\phi_{k}\right) x+\sin \left(\theta_{k}\right) \sin \left(\phi_{k}\right) y+\cos \left(\theta_{k}\right) z\right)} & \text { for }(x, y, z) \in \Omega_{k} \\
0 & \text { for }(x, y, z) \in \Omega_{k}
\end{array},\right.
$$

where $\Omega_{k}$ is the selected antenna set for user $k$. The array steering vector is given by

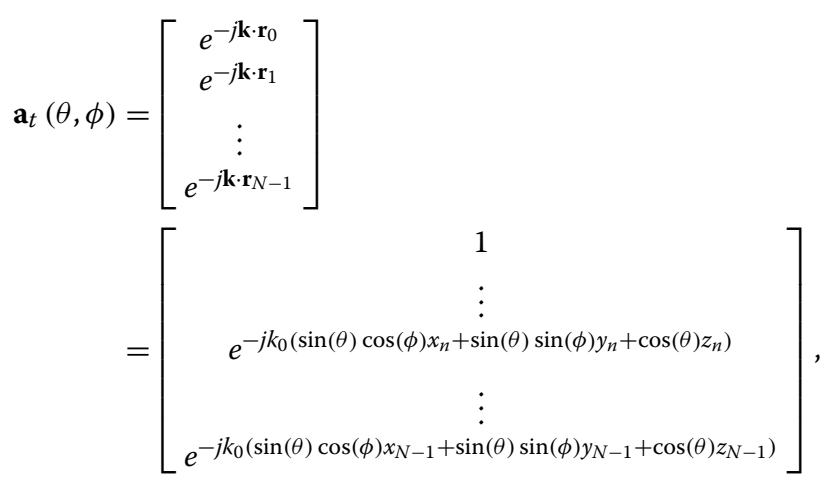

where $N=N_{x} N_{y} N_{z}$ is the number of antenna elements, and $\mathbf{k}$ is wave vector in (7), and $\mathbf{r}_{n}=\left(x_{n}, y_{n}, z_{n}\right)$ is position vector for each antenna element. The magnitude of beam pattern can be obtained by the inner product of weight vector and steering vector,

$$
\begin{aligned}
& \left|B_{k}\left(u_{x}, u_{y}, u_{z}\right)\right| \\
& =\left|\sum_{x=x_{1}^{k}} \sum_{y=y_{1}^{k}}^{x_{1}^{k}+M_{x}} \sum_{z=z_{1}^{k}}^{k} e^{j k_{0}}\left\{x d_{x}\left(u_{x}^{k}-u_{x}\right)+y d_{y}\left(u_{y}^{k}-u_{y}\right)+z d_{z}\left(u_{z}^{k}-u_{z}\right)\right\}\right| \\
& =\frac{\sin \left(\frac{M_{x} k_{0} d_{x}\left(u_{x}^{k}-u_{x}\right)}{2}\right)}{\sin \left(\frac{k_{0} d_{x}\left(u_{x}^{k}-u_{x}\right)}{2}\right)} \frac{\sin \left(\frac{M_{y} k_{0} d_{y}\left(u_{y}^{k}-u_{y}\right)}{2}\right)}{\sin \left(\frac{k_{0} d_{y}\left(u_{y}^{k}-u_{y}\right)}{2}\right)} \frac{\sin \left(\frac{M_{z} k_{0} d_{z}\left(u_{z}^{k}-u_{z}\right)}{2}\right)}{\sin \left(\frac{k_{0} d_{z}\left(u_{z}^{k}-u_{z}\right)}{2}\right)},
\end{aligned}
$$

where $u_{x}^{k}=\sin \theta_{k} \cos \phi_{k}, u_{y}^{k}=\sin \theta_{k} \sin \phi_{k}$, and $u_{z}^{k}=$ $\cos \theta_{k}$, and $\left(x_{1}^{k}, y_{1}^{k}, z_{1}^{k}\right)$ is the first element in $\Omega_{k}=$ $\left\{\left(x_{1}^{k}, y_{1}^{k}, z_{1}^{k}\right),\left(x_{1}^{k}, y_{1}^{k}, z_{1}^{k}+1\right), \cdots,\left(x_{1}^{k}+M_{x}, y_{1}^{k}+M_{y}, z_{1}^{k}+M_{z}\right)\right\}$, and $M_{x}, M_{y}$, and $M_{z}$ are the number of elements along each axis in a subset. For each axis, the first null occurred at [15]

$$
\begin{aligned}
& u_{x}=u_{x}^{k} \pm \frac{\lambda}{d_{x} M_{x}}, \\
& u_{y}=u_{y}^{k} \pm \frac{\lambda}{d_{y} M_{y}}, \\
& u_{z}=u_{z}^{k} \pm \frac{\lambda}{d_{z} M_{z}} .
\end{aligned}
$$

In the case of cuboidal array, we can express the null to null beamwidth for the fixed antenna selection as follows:

$$
\mathrm{BW}_{\mathrm{NN}}^{\mathrm{fixed}}=\frac{2 \lambda}{d_{x} M_{x}} \frac{2 \lambda}{d_{y} M_{y}} \frac{2 \lambda}{d_{z} M_{z}}
$$

If the antenna spacing is half-wavelength, (15) reduces to $\mathrm{BW}_{\mathrm{NN}}^{\text {fixed }}=\frac{64}{M}$.

\subsubsection{Random antenna selection}

Since the signal for user- $k$ is transmitted through randomly selected antenna elements, a random variable should be introduced to represent the randomness of antenna selection. The transmitted signal of an antenna element for user- $k$ is either multiplication of its weight with spread symbol or zero. If the antenna element is selected, the non-zero signal is transmitted at the element. If the element is not selected, no signal for user- $k$ is transmitted at the element. For this reason, Bernoulli random variable is applied to indicate whether an element is 
selected or not. The mean magnitude of beam pattern for random antenna selection can be written as

$$
\begin{aligned}
& \mathbb{E}\left[\left|B_{k}\left(u_{x}, u_{y}, u_{z}\right)\right|\right] \\
& =\mathbb{E}\left[\left|\sum_{x=0}^{N_{x}} \sum_{y=0}^{N_{y}} \sum_{z=0}^{N_{z}} e^{j k_{0}}\left\{x d_{x}\left(u_{x}^{k}-u_{x}\right)+y d_{y}\left(u_{y}^{k}-u_{y}\right)+z d_{z}\left(u_{z}^{k}-u_{z}\right)\right\}_{Z_{x, y, z}^{(k)} \mid}\right|\right] \\
& =\frac{M}{N} \frac{\sin \left(\frac{N_{x} k_{0} d_{x}\left(u_{x}^{k}-u_{x}\right)}{2}\right)}{\sin \left(\frac{k_{0} d_{x}\left(u_{x}^{k}-u_{x}\right)}{2}\right)} \frac{\sin \left(\frac{N_{y} k_{0} d_{y}\left(u_{y}^{k}-u_{y}\right)}{2}\right)}{\sin \left(\frac{k_{0} d_{y}\left(u_{y}^{k}-u_{y}\right)}{2}\right)} \frac{\sin \left(\frac{N_{z} k_{0} d_{z}\left(u_{z}^{k}-u_{z}\right)}{2}\right)}{\sin \left(\frac{k_{0} d_{z}\left(u_{z}^{k}-u_{z}\right)}{2}\right)},
\end{aligned}
$$

where $Z_{x, y, z}^{(k)}$ is Bernoulli random variable for the antenna element at $(x, y, z)$ denoted as $Z_{x, y, z}^{(k)} \sim \operatorname{Bern}\left(\frac{M}{N}\right)$. Similar to the case of the fixed antenna selection, the null to null beamwidth for the random antenna selection with cuboidal array can be expressed as,

$$
\mathrm{BW}_{\mathrm{NN}}^{\mathrm{random}}=\frac{2 \lambda}{d_{x} N_{x}} \frac{2 \lambda}{d_{y} N_{y}} \frac{2 \lambda}{d_{z} N_{z}} .
$$

If the antenna spacing is half-wavelength, (17) reduces to $\mathrm{BW}_{\mathrm{NN}}^{\mathrm{random}}=\frac{64}{N}$.

From (15) and (17), we can see that the random antenna selection provides a narrower beamwidth than the fixed antenna selection at the rate of $\frac{M}{N}$.

\subsection{Interference analysis}

We assume that the mmWave channel is dominated by LOS component as mentioned earlier. In Section 5, we see that the theoretical analysis can be applied to realistic mmWave channel. The normalized mmWave channel can be approximated as follows [11],

$$
\begin{aligned}
\mathbf{h} & =\sum_{p=1}^{\bar{N}} \beta_{p} \mathbf{a}_{t}\left(\theta_{p}, \phi_{p}\right) \\
& \approx \mathbf{a}_{t}\left(\theta_{L O S}, \phi_{L O S}\right),
\end{aligned}
$$

where $\mathbf{a}_{t}(\theta, \phi)$ is array steering vector.

\subsubsection{Fixed antenna selection}

To compare the interference between the two selection schemes, we first consider the fixed antenna selection scheme.

At the chip time $l$, the signal for user- $k$ transmitted from antenna element of position $(x, y, z)$ can be written as

$$
\begin{aligned}
X_{x, y, z}^{(k)}[l] & =w_{k}(x, y, z) C^{(k)}[l] s_{k} \\
& =C^{(k)}[l] s_{k} \frac{1}{M} e^{j k_{0}\left(\sin \left(\theta_{k}\right) \cos \left(\phi_{k}\right) x+\sin \left(\theta_{k}\right) \sin \left(\phi_{k}\right) y+\cos \left(\theta_{k}\right) z\right)} .
\end{aligned}
$$

The interference with the signal for user- $k$ affecting user$m$ is represented by $I_{k, m}[l]$ as follows,

$$
\begin{aligned}
I_{k, m}[l] & \\
= & \mathbf{h}_{m}^{H} \mathbf{w}_{k} x_{k}[l] \\
= & \sum_{x=x_{1}^{k}} \sum_{y=y_{1}^{k}}^{x_{1}^{k}+M_{x}} \sum_{z=z_{1}^{k}+M_{y}}^{z_{1}^{k}+M_{z}} X_{x, y, z}^{(k)}[l] \\
& \times e^{-j k_{0}\left(\sin \left(\theta_{m}\right) \cos \left(\phi_{m}\right) x+\sin \left(\theta_{m}\right) \sin \left(\phi_{m}\right) y+\cos \left(\theta_{m}\right) z\right)} \\
= & \sum_{x=x_{1}^{k}} \sum_{y=y_{1}^{k}}^{x_{1}^{k}+M_{x}} \sum_{z=z_{1}^{k}+M_{y}}^{z_{1}^{k}+M_{z}} \frac{s_{k}}{M} C^{(k)}[l] \\
& \times e^{j k_{0}}\left\{x\left(\sin \theta_{k} \cos \phi_{k}-\sin \theta_{m} \cos \phi_{m}\right)+y\left(\sin \theta_{k} \sin \phi_{k}-\sin \theta_{m} \sin \phi_{m}\right)+z\left(\cos \theta_{k}-\cos \theta_{m}\right)\right\} \\
= & \sum_{x=x_{1}^{k}+M_{x}} \sum_{y=y_{1}^{k}+M_{y}} \sum_{z=z_{1}^{k}+M_{z}} \frac{s_{k}}{M} C^{(k)}[l] e^{j\left(2 x \alpha_{k 1}+2 y \beta_{k 1}+2 z \gamma_{k 1}\right),},
\end{aligned}
$$

where

$$
\begin{aligned}
\alpha_{k m} & \triangleq \frac{1}{2} k_{0}\left(\sin \theta_{k} \cos \phi_{k}-\sin \theta_{m} \cos \phi_{m}\right), \\
\beta_{k m} & \triangleq \frac{1}{2} k_{0}\left(\sin \theta_{k} \sin \phi_{k}-\sin \theta_{m} \sin \phi_{m}\right), \\
\gamma_{k m} & \triangleq \frac{1}{2} k_{0}\left(\cos \theta_{k}-\cos \theta_{m}\right) .
\end{aligned}
$$

After despreading, the interference component in the received signal can be expressed as follows,

$$
\begin{aligned}
y_{k, m}= & \frac{1}{L} \sum_{l=1}^{L} C^{(m)}[l] I_{k, m}[l] \\
= & \frac{\rho_{k, m}}{M} s_{k} \sum_{x=x_{1}^{k}}^{x_{1}^{k}+M_{x} y_{1}^{k}+M_{y} z_{1}^{k}+M_{z}} \sum_{z=z_{1}^{k}}^{k} e^{j\left(2 x \alpha_{k m}+2 y \beta_{k m}+2 z \gamma_{k m}\right)} \\
= & \frac{\rho_{k, m}}{M} s_{k} e^{j\left\{2 x_{1}^{k} \alpha_{k m}+2 y_{1}^{k} \beta_{k m}+2 z_{1}^{k} \gamma_{k m}\right\}} \\
& \times \frac{\sin \left(M_{x} \alpha_{k m}\right)}{\sin \left(\alpha_{k m}\right)} \frac{\sin \left(M_{y} \beta_{k m}\right)}{\sin \left(\beta_{k m}\right)} \frac{\sin \left(M_{z} \gamma_{k m}\right)}{\sin \left(\gamma_{k m}\right)}
\end{aligned}
$$

where $\rho_{k, m}=\frac{1}{L} \sum_{l=1}^{L} \mathrm{C}^{(\mathrm{m})}[l] \mathrm{C}^{(\mathrm{k})}[l]$.

Since the symbol $s_{k}$ is a random variable, we can model the total interference as the sum of random variables which can be approximated as complex Gaussian distribution by central limit theorem. Therefore, we need to analyze mean and variance only. Assuming the modulation scheme as $M$-PSK, the mean of $y_{k, m}$ can be calculated as follows, 


$$
\begin{aligned}
\mathbb{E}_{\psi_{k}}\left[y_{k, m}\right]= & \frac{\rho_{k, m} \sqrt{E_{s}}}{M} \mathbb{E}_{\psi_{k}}\left[e^{\left.j\left\{\psi_{k}+2 x_{1}^{k} \alpha_{k m}+2 y_{1}^{k} \beta_{k m}+2 z_{1}^{k} \gamma_{k m}\right\}\right]}\right. \\
& \times \frac{\sin \left(M_{x} \alpha_{k m}\right)}{\sin \left(\alpha_{k m}\right)} \frac{\sin \left(M_{y} \beta_{k m}\right)}{\sin \left(\beta_{k m}\right)} \frac{\sin \left(M_{z} \gamma_{k m}\right)}{\sin \left(\gamma_{k m}\right)} \\
= & 0,
\end{aligned}
$$

where $s_{k}$ is expressed as $\sqrt{E_{s}} e^{\psi_{k}}$, and $E_{s}$ is the symbol energy, and $\psi_{k}$ is the random variable representing the phase of the symbol for user- $k$.

The variance of $y_{k, m}$ can be expressed with two components; real part and imaginary part. The variances of each part can be found as

$$
\begin{aligned}
\operatorname{var}\left[\operatorname{Re}\left\{y_{k, m}\right\}\right]= & \frac{E_{s}}{2 M^{2}} \rho_{k, m}^{2}\left(\frac{\sin \left(M_{x} \alpha_{k m}\right)}{\sin \left(\alpha_{k m}\right)}\right)^{2} \\
& \times\left(\frac{\sin \left(M_{y} \beta_{k m}\right)}{\sin \left(\beta_{k m}\right)}\right)^{2}\left(\frac{\sin \left(M_{z} \gamma_{k m}\right)}{\sin \left(\gamma_{k m}\right)}\right)^{2}, \\
\operatorname{var}\left[\operatorname{Im}\left\{y_{k, m}\right\}\right]= & \frac{E_{s}}{2 M^{2}} \rho_{k, m}^{2}\left(\frac{\sin \left(M_{x} \alpha_{k m}\right)}{\sin \left(\alpha_{k m}\right)}\right)^{2} \\
& \times\left(\frac{\sin \left(M_{y} \beta_{k m}\right)}{\sin \left(\beta_{k m}\right)}\right)^{2}\left(\frac{\sin \left(M_{z} \gamma_{k m}\right)}{\sin \left(\gamma_{k m}\right)}\right)^{2} .
\end{aligned}
$$

Since the covariance between $\operatorname{Re}\left\{y_{k, m}\right\}$ and $\operatorname{Im}\left\{y_{k, m}\right\}$ becomes zero, the total interference affecting user- $m$ can be expressed as follows,

$$
\begin{array}{r}
\sum_{\substack{k=1 \\
k \neq m}}^{K} y_{k, m} \sim \mathcal{C N}\left(0, \sum_{\substack{k=1 \\
k \neq m}}^{K} \frac{E_{s}}{M^{2}} \rho_{k, m}^{2}\left(\frac{\sin \left(M_{x} \alpha_{k m}\right)}{\sin \left(\alpha_{k m}\right)}\right)^{2}\right. \\
\left.\left(\frac{\sin \left(M_{y} \beta_{k m}\right)}{\sin \left(\beta_{k m}\right)}\right)^{2}\left(\frac{\sin \left(M_{z} \gamma_{k m}\right)}{\sin \left(\gamma_{k m}\right)}\right)^{2}\right) .
\end{array}
$$

\subsubsection{Random antenna selection}

For the random antenna selection, we can express $X_{x, y, z}^{(k)}[l]$ as follows:

$$
\begin{aligned}
& X_{x, y, z}^{(k)}[l] \\
& =C^{(k)}[l] s_{k} \frac{1}{M} e^{j k_{0}\left(\sin \left(\theta_{k}\right) \cos \left(\phi_{k}\right) x+\sin \left(\theta_{k}\right) \sin \left(\phi_{k}\right) y+\cos \left(\theta_{k}\right) z\right)} Z_{x, y, z}^{(k)} .
\end{aligned}
$$

The $I_{k, m}[l]$ can be written as

$$
\begin{aligned}
& I_{k, m}[l] \\
& \quad=\sum_{x=0}^{N_{x}-1} \sum_{y=0}^{N_{y}-1} \sum_{z=0}^{N_{z}-1} \frac{s_{k}}{M} C^{(k)}[l] Z_{x, y, z}^{(k)} e^{j\left\{2 x \alpha_{k m}+2 y \beta_{k m}+2 z \gamma_{k m}\right\}} .
\end{aligned}
$$

After despreading, the interference can be expressed as follows:

$$
\begin{aligned}
y_{k, m} & =\frac{1}{L} \sum_{l=1}^{L} C^{(m)}[l] I_{k, 1}[l] \\
& =\frac{\rho_{k, m}}{M} s_{k} \sum_{x=0}^{N_{x}-1} \sum_{y=0}^{N_{y}-1} \sum_{z=0}^{N_{z}-1} e^{j\left\{2 x \alpha_{k m}+2 y \beta_{k m}+2 z \gamma_{k m}\right\}} Z_{x, y, z}^{(k)}
\end{aligned}
$$

In contrast with the fixed antenna selection, each $y_{k, m}$ can be approximated as Gaussian random variable because it is the sum of bernoulli random variables.

First, we consider the randomness caused by random antenna selection for a given symbol. The mean of real part of (28) for a given symbol can be calculated as follows:

$$
\begin{aligned}
\mathbb{E}_{Z} & {\left[\operatorname{Re}\left\{y_{k, m}\right\} \mid \psi_{k}\right] } \\
& =\frac{\rho_{k, m} \sqrt{E_{s}}}{N} \sum_{x=0}^{N_{x}-1} \sum_{y=0}^{N_{y}-1} \sum_{z=0}^{N_{z}-1} \cos \left(\psi_{k}+2 x \alpha_{k m}+2 y \beta_{k m}+2 z \gamma_{k m}\right) \\
& =\frac{\rho_{k, m} \sqrt{E_{s}}}{2 N}\left\{\begin{array}{l}
\sum_{x=0}^{N_{x}-1} \sum_{y=0}^{N_{y}-1} \sum_{z=0}^{N_{z}-1} e^{j\left(\psi_{k}+2 x \alpha_{k m}+2 y \beta_{k m}+2 z \gamma_{k m}\right)} \\
+\sum_{x=0}^{N_{x}-1} \sum_{y=0}^{N_{y}-1} \sum_{z=0}^{N_{z}-1} e^{-j\left(\psi_{k}+2 x \alpha_{k m}+2 y \beta_{k m}+2 z \gamma_{k m}\right)}
\end{array}\right\} \\
& =\frac{\rho_{k, m} \sqrt{E_{s}}}{N} \frac{\sin \left(N_{x} \alpha_{k m}\right)}{\sin \left(\alpha_{k m}\right)} \frac{\sin \left(N_{y} \beta_{k m}\right)}{\sin \left(\beta_{k m}\right)} \frac{\sin \left(N_{z} \gamma_{k m}\right)}{\sin \left(\gamma_{k m}\right)} \cos \left(\psi_{k}\right) .
\end{aligned}
$$

Similarly, the mean of imaginary part of (28) for a given symbol can be found as

$$
\begin{aligned}
& \mathbb{E}_{Z}\left[\operatorname{Im}\left\{y_{k, m}\right\} \mid \psi_{k}\right] \\
& =\frac{\rho_{k, m} \sqrt{E_{s}}}{N} \frac{\sin \left(N_{x} \alpha_{k m}\right)}{\sin \left(\alpha_{k m}\right)} \frac{\sin \left(N_{y} \beta_{k m}\right)}{\sin \left(\beta_{k m}\right)} \frac{\sin \left(N_{z} \gamma_{k m}\right)}{\sin \left(\gamma_{k m}\right)} \sin \left(\psi_{k}\right) .
\end{aligned}
$$


The variances of real and imaginary part of (28) for a given symbol can be calculated as follows:

$$
\begin{aligned}
\operatorname{var}[ & \left.\operatorname{Re}\left\{y_{k, m}\right\} \mid \psi_{k}\right] \\
= & \frac{E_{s}(N-M)}{2 M N^{2}} \rho_{k, m}^{2} \sum_{x=0}^{N_{x}-1} \sum_{y=0}^{N_{y}-1} \sum_{z=0}^{N_{z}-1}\left\{1+\cos \left(2 \psi_{k}\right.\right. \\
& \left.\left.+4 x \alpha_{k m}+4 y \beta_{k m}+4 z \gamma_{k m}\right)\right\} \\
= & \frac{E_{s}(N-M)}{2 M N^{2}} \rho_{k, m}^{2}\left\{N+\frac{\sin \left(2 N_{x} \alpha_{k m}\right)}{\sin \left(2 \alpha_{k m}\right)} \frac{\sin \left(2 N_{y} \beta_{k m}\right)}{\sin \left(2 \beta_{k m}\right)}\right. \\
& \left.\times \frac{\sin \left(2 N_{z} \gamma_{k m}\right)}{\sin \left(2 \gamma_{k m}\right)} \cos \left(2 \psi_{k}\right)\right\},
\end{aligned}
$$

$\operatorname{var}\left[\operatorname{Im}\left\{y_{k, m}\right\} \mid \psi_{k}\right]$

$$
\begin{aligned}
= & \frac{E_{s}(N-M)}{2 M N^{2}} \rho_{k, m}^{2} \sum_{x=0}^{N_{x}-1} \sum_{y=0}^{N_{y}-1} \sum_{z=0}^{N_{z}-1}\left\{1-\cos \left(2 \psi_{k}\right.\right. \\
& \left.\left.+4 x \alpha_{k m}+4 y \beta_{k m}+4 z \gamma_{k m}\right)\right\} \\
= & \frac{E_{s}(N-M)}{2 M N^{2}} \rho_{k, m}^{2}\left\{N-\frac{\sin \left(2 N_{x} \alpha_{k m}\right)}{\sin \left(2 \alpha_{k m}\right)} \frac{\sin \left(2 N_{y} \beta_{k m}\right)}{\sin \left(2 \beta_{k m}\right)}\right. \\
& \left.\times \frac{\sin \left(2 N_{z} \gamma_{k m}\right)}{\sin \left(2 \gamma_{k m}\right)} \cos \left(2 \psi_{k}\right)\right\},
\end{aligned}
$$

where the relation $\operatorname{var}\left[Z_{x, y, z}^{(k)}\right]=\frac{M(N-M)}{N^{2}}$ is used. The correlation between real and imaginary part is calculated to obtain the covariance.
Using the second-order moment, $\mathbb{E}_{Z}\left[\left\{Z_{x, y, z}^{(k)}\right\}^{2}\right]=\frac{M}{N}$, (A) can be found as

$$
\begin{aligned}
(\mathrm{A})= & \frac{\rho_{k, m}^{2} E_{s}}{2 N M} \frac{\sin \left(2 N_{x} \alpha_{k m}\right)}{\sin \left(2 \alpha_{k m}\right)} \frac{\sin \left(2 N_{y} \beta_{k m}\right)}{\sin \left(2 \beta_{k m}\right)} \\
& \times \frac{\sin \left(2 N_{z} \gamma_{k m}\right)}{\sin \left(2 \gamma_{k m}\right)} \sin \left(2 \psi_{k}\right) .
\end{aligned}
$$

(B) is calculated as follows:

$$
\begin{aligned}
& \text { (B) }=\frac{\rho_{k, m}^{2} E_{s}}{N^{2}}\left[\left\{\sum_{x=0}^{N_{x}-1} \sum_{y=0}^{N_{y}-1} \sum_{z=0}^{N_{z}-1} \cos \left(\psi_{k}+2 x \alpha_{k m}+2 y \beta_{k m}+2 z \gamma_{k m}\right)\right\}\right. \\
& \times\left\{\sum_{\substack{x^{\prime}=0 \\
x^{\prime} \neq x}}^{N_{x}-1} \sum_{\substack{y^{\prime}=0 \\
y^{\prime} \neq y}}^{N_{y}-1} \sum_{\substack{z_{z}^{\prime}=0 \\
z^{\prime} \neq z}}^{N_{z}-1} \sin \left(\psi_{k}+2 x^{\prime} \alpha_{k m}+2 y^{\prime} \beta_{k m}+2 z^{\prime} \gamma_{k m}\right)\right\} \\
&\left.-\sum_{x=0}^{N_{x}-1} \sum_{y=0}^{N_{y}-1} \sum_{z=0}^{N_{z}-1} \frac{1}{2} \sin \left(2 \psi_{k}+4 x \alpha_{k m}+4 y \beta_{k m}+4 z \gamma_{k m}\right)\right] \\
&=\frac{\rho_{k, m}^{2} E_{s}}{N^{2}}\left[\left\{\frac{\sin \left(N_{x} \alpha_{k m}\right)}{\sin \left(\alpha_{k m}\right)} \frac{\sin \left(N_{y} \beta_{k m}\right)}{\sin \left(\beta_{k m}\right)} \frac{\sin \left(N_{z} \gamma_{k m}\right)}{\sin \left(\gamma_{k m}\right)} \cos \left(\psi_{k}\right)\right\}\right. \\
& \quad \times\left\{\frac{\sin \left(N_{x} \alpha_{k m}\right)}{\sin \left(\alpha_{k m}\right)} \frac{\sin \left(N_{y} \beta_{k m}\right)}{\sin \left(\beta_{k m}\right)} \frac{\sin \left(N_{z} \gamma_{k m}\right)}{\sin \left(\gamma_{k m}\right)} \sin \left(\psi_{k}\right)\right\} \\
&\left.-\frac{1}{2} \frac{\sin \left(2 N_{x} \alpha_{k m}\right)}{\sin \left(2 \alpha_{k m}\right)} \frac{\sin \left(2 N_{y} \beta_{k m}\right)}{\sin \left(2 \beta_{k m}\right)} \frac{\sin \left(2 N_{z} \gamma_{k m}\right)}{\sin \left(2 \gamma_{k m}\right)} \sin \left(2 \psi_{k}\right)\right] \\
&=\frac{\rho_{k, m}^{2} E_{s}}{N^{2}} \sin \left(2 \psi_{k}\right)\left[\frac{1}{2}\left(\frac{\sin \left(N_{x} \alpha_{k m}\right)}{\sin \left(\alpha_{k m}\right)}\right)^{2}\left(\frac{\sin \left(N_{y} \beta_{k m}\right)}{\sin \left(\beta_{k m}\right)}\right)^{2}\left(\frac{\sin \left(N_{z} \gamma_{k m}\right)}{\sin \left(\gamma_{k m}\right)}\right)^{2}\right. \\
&\left.-\frac{1}{2} \frac{\sin \left(2 N_{x} \alpha_{k m}\right)}{\sin \left(2 \alpha_{k m}\right)} \frac{\sin \left(2 N_{y} \beta_{k m}\right)}{\sin \left(2 \beta_{k m}\right)} \frac{\sin \left(2 N_{z} \gamma_{k m}\right)}{\sin \left(2 \gamma_{k m}\right)}\right] .
\end{aligned}
$$

Then, the covariance between the real and imaginary part for a given symbol can be presented in terms of (A) and (B),

$$
\begin{aligned}
& \mathbb{E}_{Z}\left[\operatorname{Re}\left\{y_{k, m}\right\} \operatorname{Im}\left\{y_{k, m}\right\} \mid \psi_{k}\right] \\
& =\mathbb{E}_{Z}\left[\begin{array}{c}
\left\{\begin{array}{c}
\left.\frac{\rho_{k, m} \sqrt{E_{s}}}{M} \sum_{x=0}^{N_{x}-1} \sum_{y=0}^{N_{y}-1} \sum_{z=0}^{N_{z}-1} \cos \left(\psi_{k}+2 x \alpha_{k m}+2 y \beta_{k m}+2 z \gamma_{k m}\right) Z_{x, y, z}^{(k)}\right\} \\
\times\left\{\frac{\rho_{k, m} \sqrt{E_{s}}}{M} \sum_{x=0}^{N_{x}-1} \sum_{y=0}^{N_{y}-1} \sum_{z=0}^{N_{z}-1} \sin \left(\psi_{k}+2 x \alpha_{k m}+2 y \beta_{k m}+2 z \gamma_{k m}\right) Z_{x, y, z}^{(k)}\right\}
\end{array}\right]
\end{array}\right] \\
& =\frac{\rho_{k, m}^{2} E_{s}}{M^{2}} \sum_{x=0}^{N_{x}-1} \sum_{y=0}^{N_{y}-1} \sum_{z=0}^{N_{z}-1} \frac{1}{2} \sin \left(2 \psi_{k}+4 x \alpha_{k m}+4 y \beta_{k m}+4 z \gamma_{k m}\right) \mathbb{E}_{Z}\left[\left\{Z_{x, y, z}^{(k)}\right\}^{2}\right] \\
& \text { (A) } \\
& +\frac{\rho_{k, m}^{2} E_{s}}{M^{2}} \sum_{x=0}^{N_{x}-1} \sum_{y=0}^{N_{y}-1} \sum_{z=0}^{N_{z}-1} \sum_{\substack{x^{\prime}=0 \\
x^{\prime} \neq x}}^{N_{x}-1} \sum_{\substack{y^{\prime}=0 \\
y^{\prime} \neq y}}^{N_{y}-1} \sum_{\substack{z^{\prime}=0 \\
z^{\prime} \neq z}}^{N_{z}-1}\left\{\mathbb{E}_{Z}\left[Z_{x, y, z}^{(k)}\right] \mathbb{E}_{Z}\left[Z_{x^{\prime}, y^{\prime}, z^{\prime}}^{(k)}\right]\right. \\
& \left.\times \cos \left(\psi_{k}+2 x \alpha_{k m}+2 y \beta_{k m}+2 z \gamma_{k m}\right) \sin \left(\psi_{k}+2 x^{\prime} \alpha_{k m}+2 y^{\prime} \beta_{k m}+2 z^{\prime} \gamma_{k m}\right)\right\} .
\end{aligned}
$$




$$
\begin{aligned}
& \operatorname{cov}\left[\operatorname{Re}\left\{y_{k, m}\right\}, \operatorname{Im}\left\{y_{k, m}\right\} \mid \psi_{k}\right] \\
& =(A)+(B)-\frac{\rho_{k, m}^{2} E_{s}}{2 N^{2}}\left(\frac{\sin \left(N_{x} \alpha_{k m}\right)}{\sin \left(\alpha_{k m}\right)}\right)^{2}\left(\frac{\sin \left(N_{y} \beta_{k m}\right)}{\sin \left(\beta_{k m}\right)}\right)^{2} \\
& \quad \times\left(\frac{\sin \left(N_{z} \gamma_{k m}\right)}{\sin \left(\gamma_{k m}\right)}\right)^{2} \sin \left(2 \psi_{k}\right) .
\end{aligned}
$$

Now, we consider the randomness of symbol and assume that $M$-PSK is used for modulation scheme. All symbols in the constellation have the same probability to be chosen. Therefore, the probability mass function of the random phase can be written as

$$
\operatorname{Pr}\left\{\psi_{k}\right\}=\left\{\begin{array}{cc}
\frac{1}{P} \text { for } \psi_{k}=0, \frac{2 \pi}{P}, \cdots, \frac{2 \pi(P-1)}{P} \\
0 \quad \text { otherwise }
\end{array}\right.
$$

where the $P$ is the modulation order. Taking the expectation with respect to the symbol, we can obtain the mean and variance of interference. From (29) and (30), the mean of the interference can be found as

$$
\begin{aligned}
\mathbb{E}_{\psi_{k}}\left[\mathbb{E}_{Z}\left[\operatorname{Re}\left\{y_{k, m}\right\} \mid \psi_{k}\right]\right] & =0, \\
\mathbb{E}_{\psi_{k}}\left[\mathbb{E}_{Z}\left[\operatorname{Im}\left\{y_{k, m}\right\} \mid \psi_{k}\right]\right] & =0 .
\end{aligned}
$$

From the relations in (29) and (31), the variance of real part is calculated as follows:

$$
\begin{aligned}
& \operatorname{var}\left[\operatorname{Re}\left\{y_{k, m}\right\}\right] \\
&= \mathbb{E}_{\psi_{k}}\left[\mathbb{E}_{Z}\left[\left\{\operatorname{Re}\left\{y_{k, m}\right\}\right\}^{2} \mid \psi_{k}\right]\right] \\
&= \mathbb{E}_{\psi_{k}}\left[\left[\mathbb{E}_{Z}\left[\operatorname{Re}\left\{y_{k, m}\right\} \mid \psi_{k}\right]\right]^{2}+\operatorname{var}\left[\operatorname{Re}\left\{y_{k, m}\right\} \mid \psi_{k}\right]\right] \\
&=\mathbb{E}_{\psi_{k}}\left[\frac{\rho_{k, m}^{2} E_{s}}{N^{2}}\left(\frac{\sin \left(N_{x} \alpha_{k m}\right)}{\sin \left(\alpha_{k m}\right)}\right)^{2}\left(\frac{\sin \left(N_{y} \beta_{k m}\right)}{\sin \left(\beta_{k m}\right)}\right)^{2}\right. \\
& \quad \times\left(\frac{\sin \left(N_{z} \gamma_{k m}\right)}{\sin \left(\gamma_{k m}\right)}\right)^{2} \cos ^{2}\left(\psi_{k}\right)+\frac{E_{s}(N-M)}{2 M N^{2}} \rho_{k, m}^{2} \\
&\left.\quad \times\left\{N+\frac{\sin \left(2 N_{x} \alpha_{k m}\right)}{\sin \left(2 \alpha_{k m}\right)} \frac{\sin \left(2 N_{y} \beta_{k m}\right)}{\sin \left(2 \beta_{k m}\right)} \frac{\sin \left(2 \gamma_{z k m}\right)}{\sin \left(2 \gamma_{k m}\right)} \cos \left(2 \psi_{k}\right)\right\}\right] \\
&=\frac{\rho_{k, m}^{2} E_{s}}{2 N^{2}}\left(\frac{\sin \left(N_{x} \alpha_{k m}\right)}{\sin \left(\alpha_{k m}\right)}\right)^{2}\left(\frac{\sin \left(N_{y} \beta_{k m}\right)}{\sin \left(\beta_{k m}\right)}\right)^{2}\left(\frac{\sin \left(N_{z} \gamma_{k m}\right)}{\sin \left(\gamma_{k m}\right)}\right)^{2} \\
& \quad+\frac{E_{s}(N-M)}{2 M N} \rho_{k, m}^{2} .
\end{aligned}
$$

In the same way, the variance of imaginary part also can be found from the relations in (30) and (32).

$$
\begin{aligned}
\operatorname{var}\left[\operatorname{Im}\left\{y_{k, m}\right\}\right]= & \frac{\rho_{k, m}^{2} E_{s}}{2 N^{2}}\left(\frac{\sin \left(N_{x} \alpha_{k m}\right)}{\sin \left(\alpha_{k m}\right)}\right)^{2}\left(\frac{\sin \left(N_{y} \beta_{k m}\right)}{\sin \left(\beta_{k m}\right)}\right)^{2} \\
& \times\left(\frac{\sin \left(N_{z} \gamma_{k m}\right)}{\sin \left(\gamma_{k m}\right)}\right)^{2}+\frac{E_{s}(N-M)}{2 M N} \rho_{k, m}^{2} .
\end{aligned}
$$

From (36), the covariance between real and imaginary part is calculated as follows:

$$
\operatorname{cov}\left[\operatorname{Re}\left\{y_{k, m}\right\}, \operatorname{Im}\left\{y_{k, m}\right\}\right]=0 .
$$

From the relation $y_{k, m}=\operatorname{Re}\left\{y_{k, m}\right\}+j \operatorname{Im}\left\{y_{k, m}\right\}$, the mean and variance of $y_{k, m}$ are obtained as follows:

$$
\mathbb{E}\left[y_{k, m}\right]=0,
$$

$$
\begin{aligned}
\operatorname{var}\left[y_{k, m}\right]= & \frac{\rho_{k, m}^{2} E_{s}}{N^{2}}\left(\frac{\sin \left(N_{x} \alpha_{k m}\right)}{\sin \left(\alpha_{k m}\right)}\right)^{2}\left(\frac{\sin \left(N_{y} \beta_{k m}\right)}{\sin \left(\beta_{k m}\right)}\right)^{2} \\
& \times\left(\frac{\sin \left(N_{z} \gamma_{k m}\right)}{\sin \left(\gamma_{k m}\right)}\right)^{2}+\frac{E_{s}(N-M)}{M N} \rho_{k, m}^{2} .
\end{aligned}
$$

In (43), the second term comes from the randomness of each part of $y_{k, m}$ for given $\psi_{k}$, i.e., $\operatorname{var}\left[\operatorname{Re}\left\{y_{k, m}\right\} \mid \psi_{k}\right]$ and $\operatorname{var}\left[\operatorname{Im}\left\{y_{k, m}\right\} \mid \psi_{k}\right]$ as shown in (39) and (40). However, as other users' positions are closer to the desired user, these randomness caused by random antenna selection can be ignored because of high correlation of received signals. In other words, as shown in Fig. 5, the randomness of antenna selection only appears in the region of side lobes, and there is no randomness in the region of main lobe. For this reason, in the case of highly correlated users, we have to ignore the effect of random selection in (43). For example of ULA with $N$ elements, since the phase difference between adjacent antenna elements is $2 \gamma_{k, m}$, the maximum phase difference from other antenna elements is $(N-1) 2 \gamma_{k, m}$. If $(N-1) 2 \gamma_{k, m}$ is less than $\pi$, the signals cannot be canceled to some extent but be reinforced by one-directional phases (either all positive phases or all negative phases). Since the terms related to the random selection are (31) and (32) which result in (39) and (40), we ignore $\frac{E_{s}(N-M)}{M N} \rho_{k, m}^{2}$ in (43) to consider the case of highly correlated signal. As a result, we give the final expression for $y_{k, m}$ as follows: 


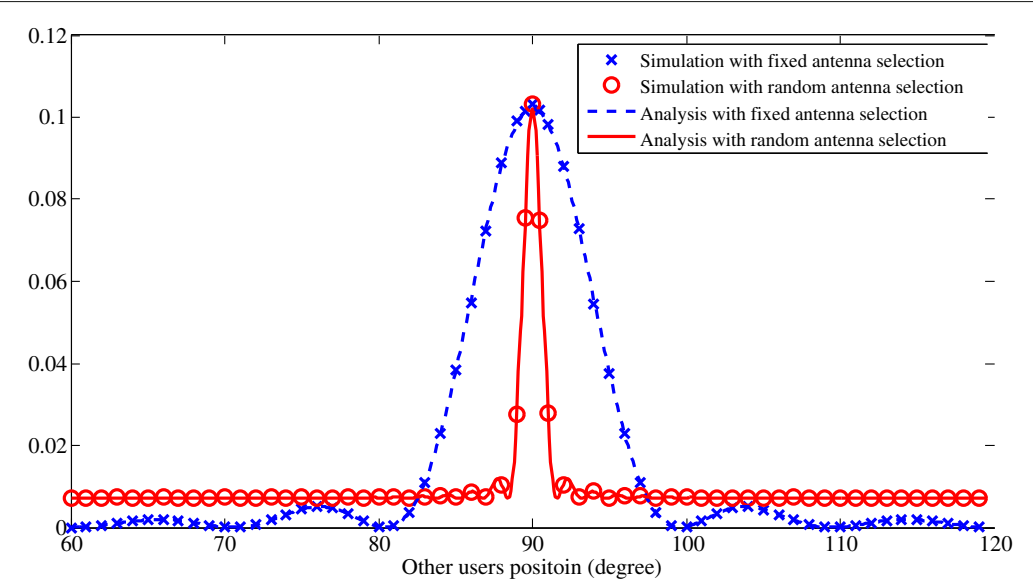

Fig. 5 The variance of total interference to a user located at $90^{\circ}: M=12, K=6$

$$
\begin{gathered}
y_{k, m} \sim \mathcal{C N}\left(0, \frac{\rho_{k, m}^{2} E_{s}}{N^{2}}\left(\frac{\sin \left(N_{x} \alpha_{k m}\right)}{\sin \left(\alpha_{k m}\right)}\right)^{2}\left(\frac{\sin \left(N_{y} \beta_{k m}\right)}{\sin \left(\beta_{k m}\right)}\right)^{2}\right. \\
\left.\left(\frac{\sin \left(N_{z} \gamma_{k m}\right)}{\sin \left(\gamma_{k m}\right)}\right)^{2}+T_{k, m} \frac{E_{s}(N-M)}{M N} \rho_{k, m}^{2}\right)
\end{gathered}
$$

where

$$
\begin{aligned}
& T_{k, m} \\
& =\left\{\begin{array}{ll}
0 & \text { for }\left|\left(N_{x}-1\right) 2 \alpha_{k m}\right| \leq \pi \text { and }\left|\left(N_{y}-1\right) 2 \beta_{k m}\right| \leq \pi \text { and }\left|\left(N_{z}-1\right) 2 \gamma_{k m}\right| \leq \pi \\
1 & \text { otherwise }
\end{array},\right.
\end{aligned}
$$

and the total interference affecting user- $m$ can be expressed as $\sum_{\substack{k=1 \\ k \neq m}}^{K} y_{k, m}$.

In Fig. 5, we verify the mathematical results in (25) and (44) with simulations by assuming ULA. We can see that the random antenna selection provides a narrower beamwidth than the fixed antenna selection. In other words, the random antenna selection has lower interference than the fixed antenna selection in the range of $83^{\circ} \sim 97^{\circ}$. This result implies that the random antenna selection mitigates the inter-user interference where the interference cannot be managed effectively by beamforming. Also, the random antenna selection can provide the fairness by reducing the interference to closer users and by allowing a small amount of interference to farther users who can be tolerant of the interference. As a result, the random antenna selection mitigates the inter-user interference, and this is directly related with the beamwidth analysis in (15) and (17).

\subsection{Bit error rate}

Assuming that QPSK is used for the modulation scheme, the expressions for theoretical bit error rate (BER) for fixed antenna selection is given by,

$$
\begin{aligned}
& \mathrm{P}_{\mathrm{b}}^{\text {fixed }} \\
& =Q\left(\frac{d}{2 \sigma}\right) \\
& =Q\left(\sqrt{\frac{E_{b}}{\frac{N_{0}}{2}+\frac{P_{I}}{2}}}\right) \\
& =Q\left(\sqrt{\frac{N_{0}}{2 E_{b}}+\frac{1}{M^{2}} \sum_{\substack{k=1 \\
k \neq m}}^{K} \rho_{k, m}^{2}\left(\frac{\sin \left(M_{x} \alpha_{k m}\right)}{\sin \left(\alpha_{k m}\right)}\right)^{2}\left(\frac{\sin \left(M_{y} \beta_{k m}\right)}{\sin \left(\beta_{k m}\right)}\right)^{2}\left(\frac{\sin \left(M_{z} \gamma_{k m}\right)}{\sin \left(\gamma_{k m}\right)}\right)^{2}}\right),
\end{aligned}
$$

where the interference is considered as noise, and $P_{I}$ and $N_{0}$ are the total interference power and noise power, respectively, and $d=2 \sqrt{E_{b}}$ is the minimum distance of QPSK, and $E_{b}$ is the bit energy. Theoretical BER for random antenna selection can be obtained as

$$
\mathrm{P}_{\mathrm{b}}^{\mathrm{random}}=Q\left(\sqrt{\frac{1}{\frac{N_{0}}{2 E_{b}}+\sum_{\substack{k=1 \\ k \neq m}}^{K}\left\{\frac{\rho_{k, m}^{2}}{N^{2}}\left(\frac{\sin \left(N_{x} \alpha_{k m}\right)}{\sin \left(\alpha_{k m}\right)}\right)^{2}\left(\frac{\sin \left(N_{y} \beta_{k m}\right)}{\sin \left(\beta_{k m}\right)}\right)^{2}\left(\frac{\sin \left(N_{z} \gamma_{k m}\right)}{\sin \left(\gamma_{k m}\right)}\right)^{2}+T_{k, m} \frac{(N-M)}{M N} \rho_{k, m}^{2}\right\}}}\right) .
$$




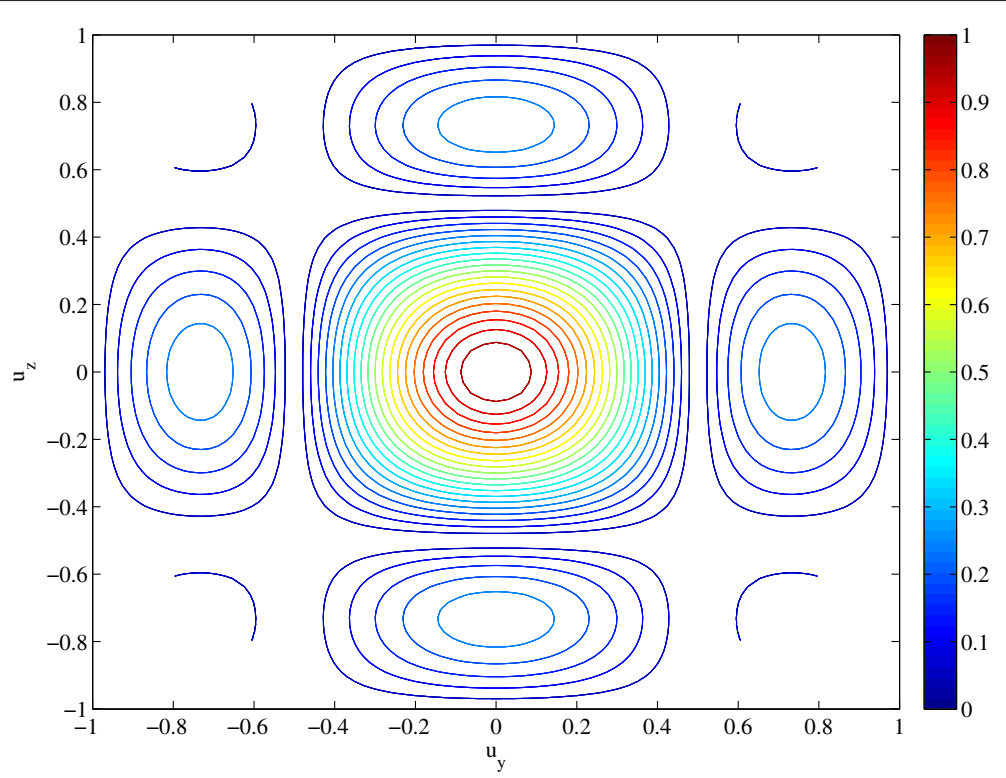

Fig. 6 Contour plot of magnitude of beam pattern for fixed antenna selection with UPA :M=16,K=6

\section{Simulation results}

In this section, we give the simulation results for the beamwidth comparison and BER performances. In our simulations, we focus on the effect of the random antenna selection in the system with only $K$ RF chains as described in Fig. 1. Therefore, we compare the performances of the proposed random antenna selection with the fixed antenna selection which is conventional in the system with sub-array beamforming structure [22, 23].

\subsection{Beamwidth}

In the simulations, we assume that the UPA is placed on yz-plane, and the array is steered to $(\theta, \phi)=\left(90^{\circ}, 0^{\circ}\right)$, and $K=6, M_{y}=4, M_{z}=4, N_{y}=12, N_{z}=8, d_{y}=$ $d_{z}=\lambda / 2$. Figure 6 and 7 show the magnitude contour of beam patterns in $\left(u_{y}, u_{z}\right)$ space. We can see that the random antenna selection gives narrower beamwidth than fixed antenna selection, and the theoretical null-tonull beamwidth (15) and (17) are well matched with the

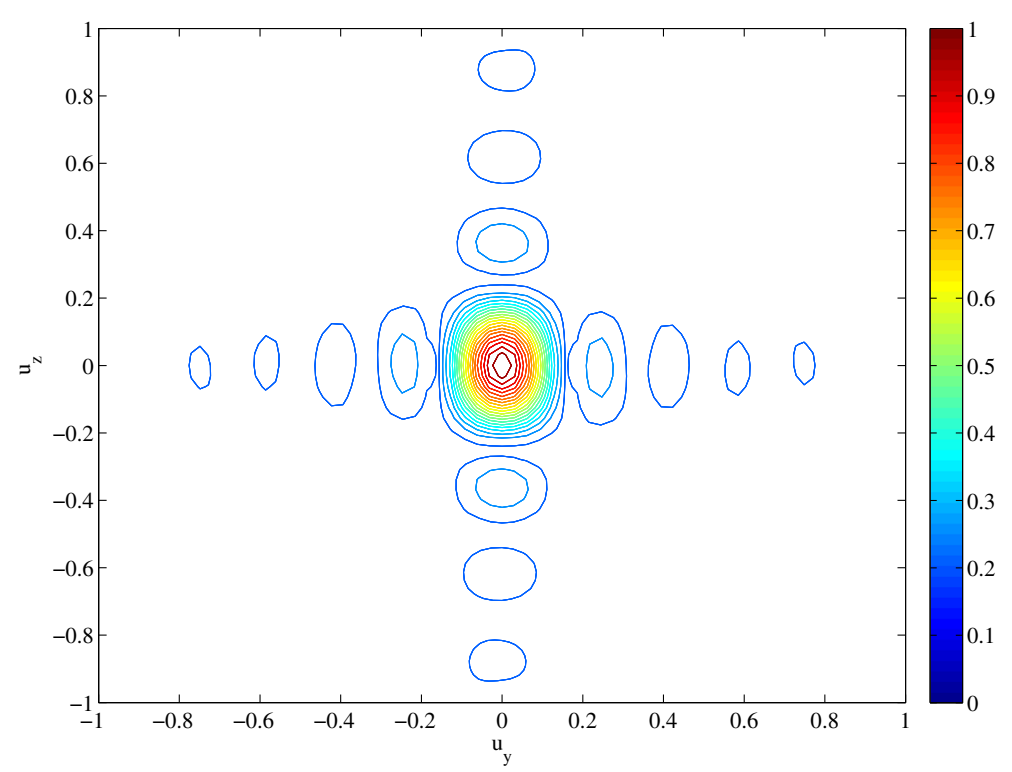

Fig. 7 Contour plot of magnitude of average beam pattern for random antenna selection with UPA :M=16,K=6 
simulation results. The first null points for fixed antenna selection occurred at $u_{y}= \pm \frac{1}{2}$ and $u_{z}= \pm \frac{1}{2}$, which results in $\mathrm{BW}_{N N}^{\mathrm{fixed}}=1$. On the other hand, the first null points for random antenna selection occurred at $u_{y}= \pm \frac{1}{6}$ and $u_{z}= \pm \frac{1}{4}$, which results in $\mathrm{BW}_{N N}^{\text {random }}=\frac{1}{6}$. Therefore, the null-to-null beamwidth for random antenna selection is six times as narrow as that for fixed antenna selection. We can see that the area of main lobes in Fig. 6 is about six times as large as that in Fig. 7. From the results, we can infer that the random antenna selection can reduce the interference with the signals for other users.

\subsection{Bit error rate}

In the simulations, we assume that user- 1 is located at $(\theta, \phi)=\left(90^{\circ}, 0^{\circ}\right)$, and other users are located at the positions with some offset degrees from user-1. The UPA lied on the $y z$-plane with $d_{y}=d_{z}=\lambda / 2$ is used. The number of multi-path components is set to be $\bar{N}=30$, and we use Gold code of length 15 for spread code and QPSK for modulation scheme. The transmitted power for each user is normalized to unity. The number of users is set to be $K=14$, and the users are located with uniform offset of $2^{\circ}$ in both elevation and azimuth angle. From the expression in (46) and (47), the theoretical BER for user-1 can be calculated as follows:

$$
\begin{aligned}
& \mathrm{P}_{\mathrm{b}}^{\text {fixed }} \\
& =Q\left(\sqrt{\frac{1}{\frac{N_{0}}{2 E_{b}}+\frac{1}{225 M^{2}} \sum_{k=2}^{14}\left(\frac{\sin \left(M_{y} \beta_{k 1}\right)}{\sin \left(\beta_{k 1}\right)}\right)^{2}\left(\frac{\sin \left(M_{z} \gamma_{k 1}\right)}{\sin \left(\gamma_{k 1}\right)}\right)^{2}}}\right),
\end{aligned}
$$

$$
\begin{aligned}
& \mathrm{P}_{\mathrm{b}}^{\text {random }} \\
& =Q\left(\sqrt{\frac{1}{\frac{N_{0}}{2 E_{b}}+\frac{1}{225 N^{2}} \sum_{k=2}^{14}\left\{\left(\frac{\sin \left(N_{y} \beta_{k 1}\right)}{\sin \left(\beta_{k 1}\right)}\right)^{2}\left(\frac{\sin \left(N_{z} \gamma_{k 1}\right)}{\sin \left(\gamma_{k 1}\right)}\right)^{2}+13 N T_{k, 1}\right\}}}\right) .
\end{aligned}
$$

First, we consider the LOS only channel in order to verify the theoretical analysis. In Fig. 8, $\rho_{L O S}$ is set to be 1 so that the channel is normalized to unity, and NLOS path does not exist. We can see that the random selection has SNR gain of $2.5 \mathrm{~dB}$ for $M=16$, and $4 \mathrm{~dB}$ for $M=4$ at BER of $10^{-4}$. The smaller the number of antenna elements, the larger the SNR gain becomes. Intuitively, the difference of effective array size between random selection and fixed selection for $M=4$ is larger than that for $M=16$. Note that the performance differences between $M=4$ and $M=16$ are from the beamforming gain due to the number of total antenna elements. Also, we can see

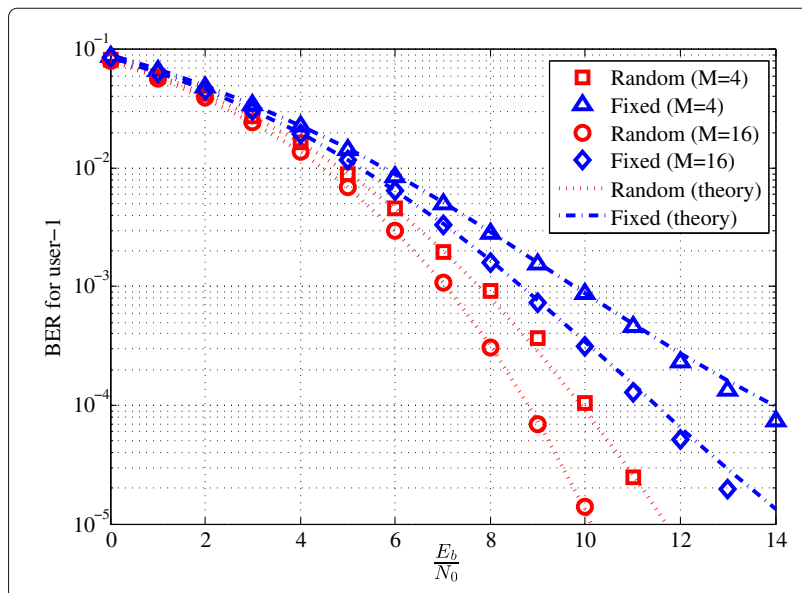

Fig. 8 BER for user-1 in LOS only channel

that the theoretical BER curves are well matched with the simulated curves. In contrast with random selection, the number of random variables summed together $(K=14)$ is not large enough in the fixed selection which gives a slight inaccuracy.

Now, we provide the BER simulation in mmWave channel model including the NLOS links. In [21], the urban outdoor mmWave channel measurements of time delay spread and path loss are provided for 38 and $60 \mathrm{GHz}$ channel. For $38 \mathrm{GHz}$ channel, the path loss difference between LOS and NLOS links is $18 \mathrm{~dB}$ at $30 \mathrm{~m}$ and $21 \mathrm{~dB}$ at $50 \mathrm{~m}$ from transmitter. The $60 \mathrm{GHz}$ channel also has similar behavior with $38 \mathrm{GHz}$. The LOS path has no resolvable multi-path, and the mean of RMS delay spread for NLOS link is $23.6 \mathrm{~ns}$ for $38 \mathrm{GHz}$ and $7.4 \mathrm{~ns}$ for $60 \mathrm{GHz}$, and the maximum RMS delay spread for NLOS link is $122 \mathrm{~ns}$ for $38 \mathrm{GHz}$ and $36.6 \mathrm{~ns}$ for $60 \mathrm{GHz}$. We can see that the delay spread for mmWave is very small especially for $60 \mathrm{GHz}$. To understand the effect of NLOS power, we assume that the number of resolvable path of NLOS is one which results in $\tau_{p}=\bar{\tau}_{r m s}$. Considering the $38 \mathrm{GHz}$ channel, $\bar{\tau}_{r m s}$ is set to be 23.6 ns. Figure 9 shows the effect of NLOS power, where $M=16$. When the path loss difference between LOS and NLOS links is larger than $16 \mathrm{~dB}$, the BER performance becomes almost the same with the LOS only channel. Since the path loss difference between LOS and NLOS links in mmWave is larger than $16 \mathrm{~dB}$ at more than $30 \mathrm{~m}$ [21], our theoretical analysis of LOS only channel can be accurately applied to the realistic mmWave channel. Moreover, we can infer that the random antenna selection has better performance than the fixed antenna selection even if the NLOS power becomes strong.

In Fig. 10, we compare the performances of the antenna selection schemes with that of digital-analog hybrid precoding technique which has $N$ antennas and $K$ RF chains. For the multi-user hybrid beamforming system, the two- 


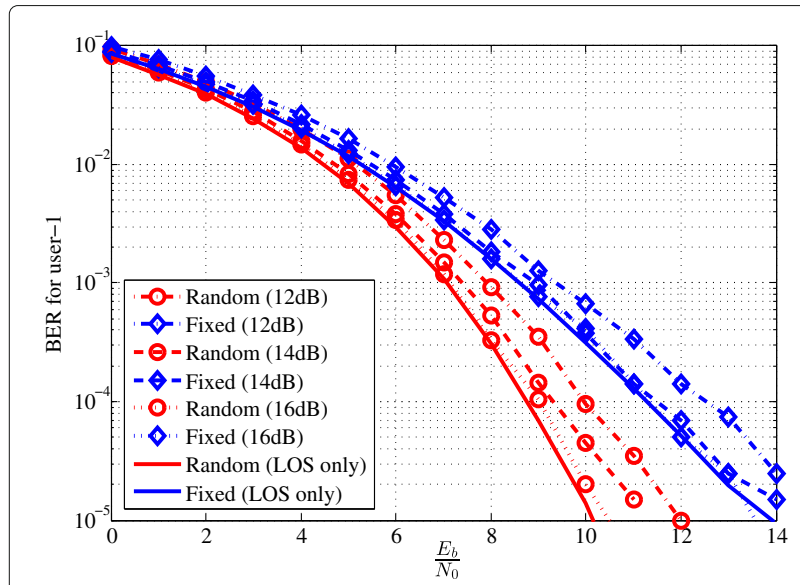

Fig. 9 BER for user-1 in mmWave channel for different NLOS power

stage beamforming technique [24] is applicable. In the first stage, we use the same beamforming vectors with the fixed selection scheme to consider the sub-array structure. Assuming that the effective channel which is the product of the channel matrix and the RF beamforming matrix is known at the transmitter, the baseband beamforming matrix is constructed as zero-forcing precoder at the second stage. We assume the same simulation parameters with Fig. 8 except for the number of antennas. We see that the antenna selection schemes with spreading code outperform the hybrid beamforming scheme when the total number of antennas $N$ is equal to $M K=504$ or $M K=896$. In case of $M K=1400$, the hybrid beamforming scheme outperforms the antenna selection schemes. Since it is not easy to implement a thousand of antennas in practical systems, the antenna selection scheme with spreading code is more attractable than the hybrid beamforming scheme in terms of BER performance at the expense of time resources.

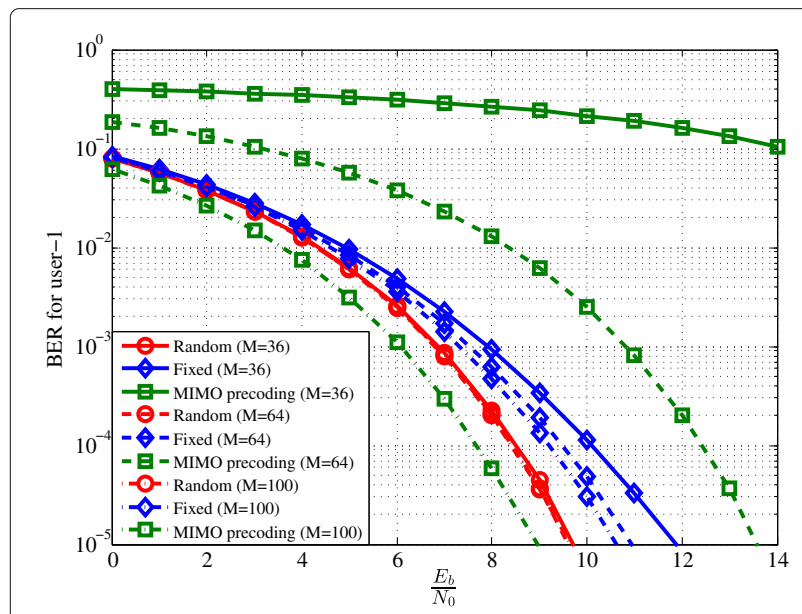

Fig. 10 BER comparison with digital-analog hybrid precoding scheme

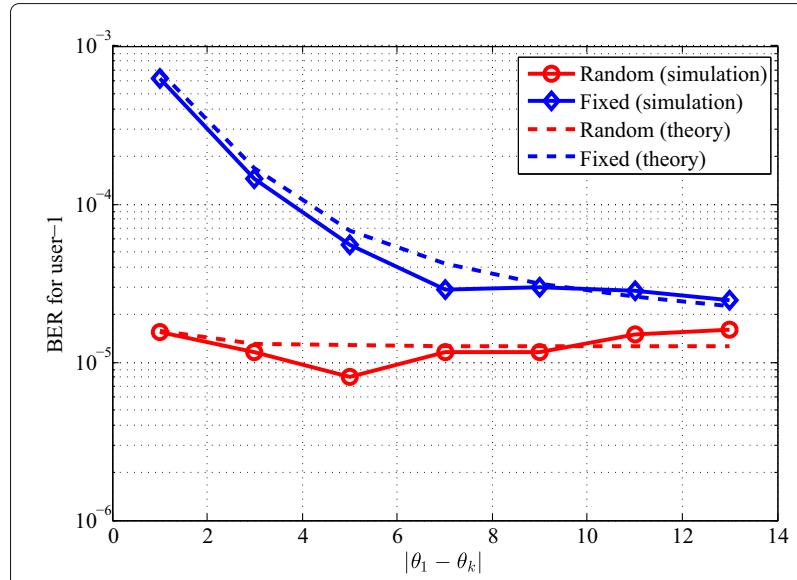

Fig. $11 \mathrm{BER}$ for user-1 versus user distribution with UPA :M $=16$, $K=14, E_{b} / N_{0}=8 \mathrm{~dB}$

In addition, we consider the effect of the position distribution of users. Figure 11 shows the BER versus user distribution for UPA. We can see that the advantage of random antenna selection becomes large when other users are located near the user- 1 . If the positions of other users are far from the user-1, the performance gap becomes small. The results for ULA is provided in Fig. 12. In contrast with UPA which conduct 3D beamforming, the ULA on $\mathrm{z}$-axis cannot distinguish the azimuth angles. Therefore, the random antenna selection with ULA has smaller performance gain than that with UPA.

\section{Conclusions}

In this paper, we have proposed the random antenna selection scheme to mitigate the inter-user interference in multi-user mmWave beamforming systems. The prime advantage of this scheme is that it expands the effective array size to make a narrow beamwidth which reduces

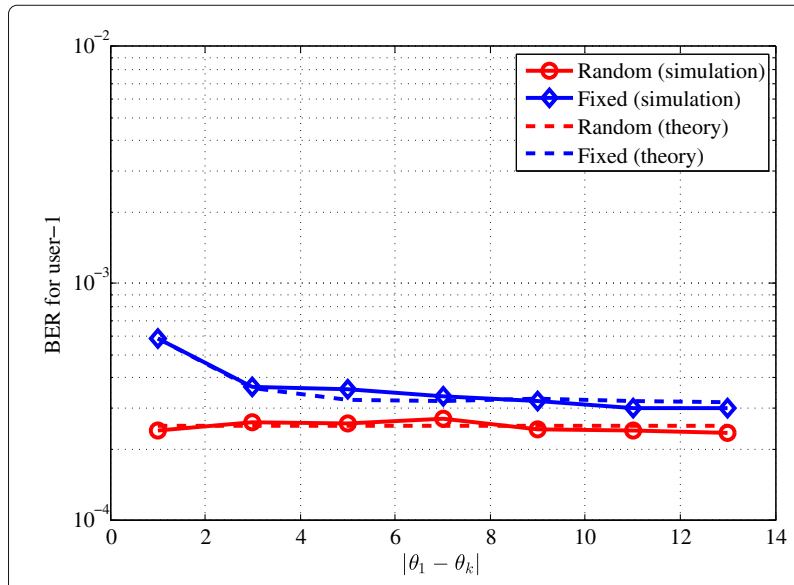

Fig. 12 BER for user- 1 versus user distribution with ULA :M $=16$, $K=14, E_{b} / N_{0}=8 \mathrm{~dB}$ 
the overlapped area between the beams for each user. The beamwidth of the random antenna selection is $\frac{M}{N}$ times as narrow as the beamwidth of the conventional fixed antenna selection. We analyze the amount of interference for each selection scheme using Gaussian approximation and compare it with the simulation results in realistic mmWave channel. The advantage of random antenna selection can be well applied to mmWave 3D beamforming systems using massive antenna array.

\section{Acknowledgements}

This research was supported by Basic Science Research Program through the National Research Foundation of Korea(NRF) funded by the Ministry of Science, ICT \& Future Planning(2017R1 A2B4009853). This work was also supported by the Institute for Information \& communications Technology Promotion (IITP) grant funded by the Korea government (MSIP) (2017-0-00765).

\section{Competing interests}

The authors declare that they have no competing interests.

\section{Publisher's Note}

Springer Nature remains neutral with regard to jurisdictional claims in published maps and institutional affiliations.

\section{Author details}

${ }^{1}$ Agency for Defense Development (ADD), P.O. Box 35, Daejeon 305-600, Republic of Korea. ${ }^{2}$ School of Electrical Engineering, Korea Advanced Institute of Science and Technology (KAIST), Daehak-ro 291, Daejeon 305-338, Republic of Korea.

Received: 24 April 2015 Accepted: 21 April 2017

Published online: 12 May 2017

\section{References}

1. SK Yong, CC Chong, An overview of multigigabit wireless through millimeter wave technology: potentials and technical challenges. EURASIP J. Wirel. Commun. Netw. 2007(078907) (2006). doi:10.1155/2007/78907

2. Z Pi, F Khan, An introduction to millimeter-wave mobile broadband systems. IEEE Commun. Mag. 49(6), 101-107 (2011)

3. DJ Love, RW Heath, T Strohmer, Grassmannian beamforming for multiple-input multiple-output wireless systems. IEEE Trans. Inform. Theory. 49(10), 2735-2747 (2003)

4. J Wang, Z Lan, C Pyo, T Baykas, C Sum, M Rahman, J Gao, R Funada, F Kojima, H Harada, et al., Beam codebook based beamforming protocol for multi-Gbps millimeter-wave WPAN systems. IEEE J. Sel. Areas Commun. 27(8), 1390-1399 (2009)

5. S-H Tsai, Equal gain transmission with antenna selection in MIMO communication. IEEE Trans. Wireless Commun. 10(5), 1470-1479 (2011)

6. DJ Love, RW Heath, Equal gain transmission in multiple-input multiple-output wireless systems. IEEE Trans. Commun. 51(7), 1102-1110 (2003)

7. OE Ayach, S Rajagopal, S Abu-Surra, Z Pi, RW Heath, Spatially sparse precoding in millimeter wave MIMO systems. IEEE Trans. Wireless Commun. 13(3), 1499-1513 (2014)

8. G Kwon, Y Shim, H Park, HM Kwon, in 2014 IEEE 80th Vehicular Technology Conference (VTC2014-Fall). Design of millimeter wave hybrid beamforming systems (IEEE, Vancouver, 2014), pp. 1-5. doi:10.1109/NTCFall.2014.6965933

9. G Kwon, H Park, in 2015 IEEE Global Communications Conference (GLOBECOM). An efficient hybrid beamforming scheme for sparse millimeter wave channel (IEEE, San Diego, 2015), pp. 1-6. doi:10.1109/GLOCOM.2015.7417344

10. TE Bogale, LB Le, A Haghighat, L Vandendorpe, On the number of RF chains and phase shifters, and scheduling design with hybrid analog-digital beamforming. IEEE Trans. Wireless Commun. 15(5), 3311-3326 (2016)
11. N Valliappan, A Lozano, RW Heath, Antenna subset modulation for secure millimeter-wave wireless communication. IEEE Trans. Commun. 61(8), 3231-3245 (2013)

12. BM Lim, K Ahn, H Kim, H Park, GT Gil, in 2010 IEEE 71st Vehicular Technology Conference. Improved user scheduling algorithms for codebook based MIMO precoding schemes (IEEE, Taipei, 2010), pp. 1-5. doi:10.1109/NETECS.2010.5493763

13. QH Spencer, AL Swindlehurst, M Haardt, Zero-forcing methods for downlink spatial multiplexing in multiuser MIMO channels. IEEE Trans. Signal Process. 52(2), 461-471 (2004)

14. C Windpassinger, RFH Fischer, T Vencel, JB Huber, Precoding in multi-antenna and multi-user communications. IEEE Trans. Wireless Commun. 3(4), 1305-1316 (2004)

15. HLV Trees, Optimum array processing: part IV of detection, estimation, and modulation theory. (Wiley-Interscience, New York, 2002)

16. S Hur, T Kim, DJ Love, JV Krogmeier, TA Thomas, A Ghosh, Millimeter wave beamforming for wireless backhaul and access in small cell networks. IEEE Trans. Commun. 61(10), 4391-4403 (2013)

17. A Alkhateeb, OE Ayach, G Leus, RW Heath, Channel estimation and hybrid precoding for millimeter wave cellular systems. IEEE J. Sel. Top. Signal Process. 8(5), 831-846 (2014)

18. J Lee, G-T Gil, YH Lee, in 2014 IEEE Global Communications Conference. Exploiting spatial sparsity for estimating channels of hybrid MIMO systems in millimeter wave communications (IEEE, Austin, 2014), pp. 3326-3331. doi:10.1109/GLOCOM.2014.7037320

19. P Almers, E Bonek, A Burr, N Czink, M Debbah, V Degli-Esposti, H Hofstetter, P Kyö, D Laurenson, G Matz, et al., Survey of channel and radio propagation models for wireless MIMO systems. EURASIP J. Wirel. Commun. Netw. 2007 2007(019070) (2007). doi:10.1155/2007/19070

20. M Steinbauer, AF Molisch, E Bonek, The double-directional radio channel. IEEE Antennas Propag. Mag. 43(4), 51-63 (2001)

21. TS Rappaport, E Ben-Dor, JN Murdock, Y Qiao, in 2012 IEEE International Conference on Communications (ICC). $38 \mathrm{GHz}$ and $60 \mathrm{GHz}$ angle-dependent propagation for cellular \& peer-to-peer wireless communications (IEEE, Ottawa, 2012), pp. 4568-4573. doi:10.1109/ICC.2012.6363891

22. OE Ayach, RW Heath, S Rajagopal, Z Pi, in 2013 IEEE Global Communications Conference (GLOBECOM). Multimode precoding in millimeter wave MIMO transmitters with multiple antenna sub-arrays, (2013), pp. 3476-3480. doi:10.1109/GLOCOM.2013.6831611

23. W Roh, JY Seol, J Park, B Lee, J Lee, Y Kim, J Cho, K Cheun, F Aryanfar, Millimeter-wave beamforming as an enabling technology for $5 \mathrm{G}$ cellular communications: theoretical feasibility and prototype results. IEEE Commun. Mag. 52(2), 106-113 (2014). doi:10.1109/MCOM.2014.6736750

24. A Alkhateeb, G Leus, RW Heath, Limited feedback hybrid precoding for multi-user millimeter wave systems. IEEE Trans. Wireless Commun. 14(11), 6481-6494 (2015)

\section{Submit your manuscript to a SpringerOpen ${ }^{\circ}$ journal and benefit from:}

- Convenient online submission

- Rigorous peer review

- Immediate publication on acceptance

- Open access: articles freely available online

- High visibility within the field

- Retaining the copyright to your article

Submit your next manuscript at $\boldsymbol{s p r i n g e r o p e n . c o m ~}$ 